\title{
Deformation and breakup of single drop in laminar and transitional jet flows
}

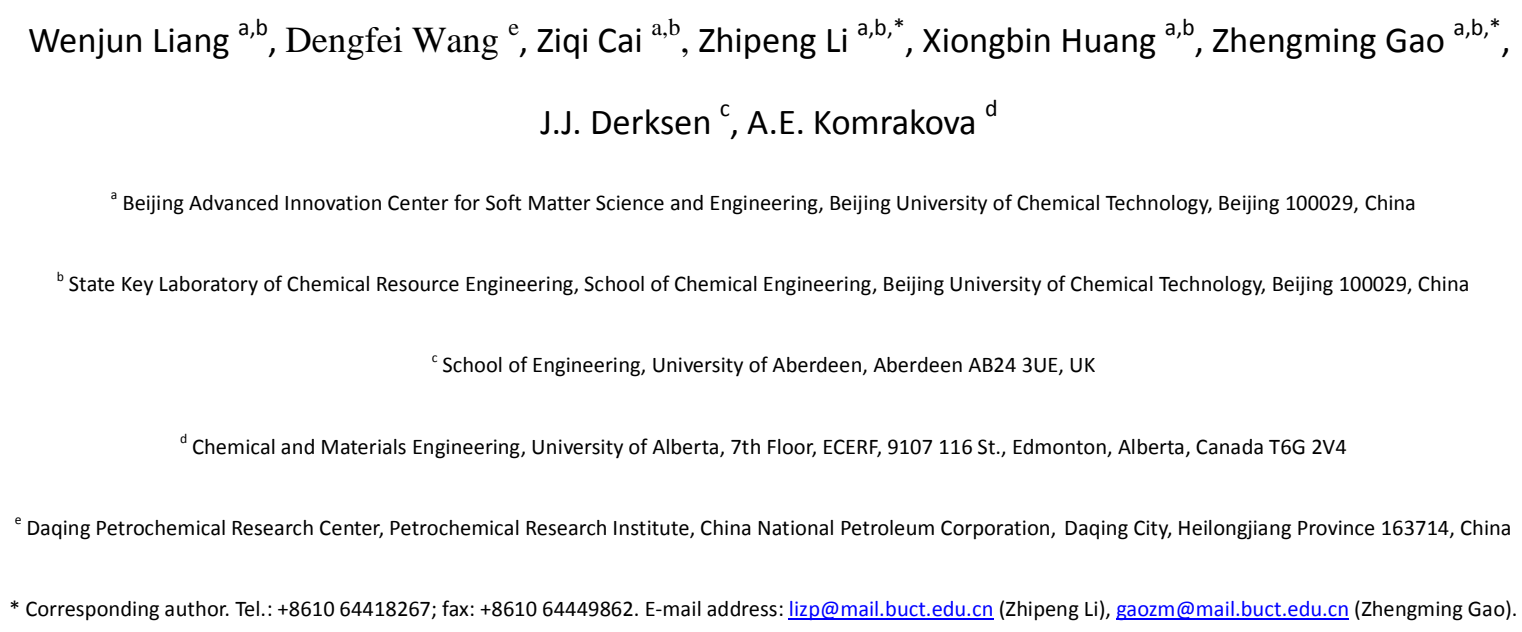

\section{Abstract:}

Liquid drops were released in laminar and transitional jet flows to investigate their deformation and breakup characteristics. Silicone oil and deionized water were the dispersed phase and continuous phase, respectively. Calibration experiments of oil drops rising in quiescent ambient water were performed to benchmark the experimental system and the image processing method. In jet flow, drop breakup probability, breakup time, and the characteristics of daughter drops were investigated in detail. To address the underlying mechanisms of the drop breakup, visualization experiments and two-dimensional particle image velocimetry (PIV) experiments of the single-phase jet flow were performed. Visualization experiments show that the jet flow changes from laminar to transitional in the Reynolds number interval between 1283 and 1610. Critical capillary and Weber numbers for drop breakup were estimated based on the mean flow velocity and mean deformation and were found to be of the order of 0.2 and 30 respectively for this particular flow system.

Keywords: drop breakup; jet flow; PIV; quantitative flow visualization; breakup mechanism

\section{Highlights:}

1) Quantitative visualization of drop deformation and breakup in jet flow was performed

2) Initial breakup/breakup cascade probability critically depend on jet Reynolds number

3) The critical conditions for drop breakup in transitional jet flow were quantified 


\section{Introduction}

Many industrial applications involve liquid-liquid dispersions and emulsions: petrochemicals (crude oil/water emulsions and oil spills in water), pharmaceuticals (lipid emulsions and anesthetics), food (salad dressings and beverages), and cosmetics (creams and lotions) [1]. The properties and behaviors of these systems depend critically on the drop size distribution (DSD) of the dispersed phase. The DSD is a result of a complex combination of simultaneously occurring processes at the level of individual drops [2], the size of which ranges from less than a micrometer to several millimeters. Therefore, a fundamental understanding of the events at the microscopic scale is required to control the macroscopic state of the dispersion. The existing models of drop breakup involve numerous assumptions and simplifications of these events (e.g. spherical drops, binary breakup, collision of a single drop with an eddy) and so are limited in their ability to reliably predict the DSD as a function of fluid properties and process conditions.

Experiments on the behavior of liquid-liquid systems under varying flow conditions provide essential insights which advance breakup models. Despite broad applicability and industrial relevance of liquid-liquid dispersions, the number of published experimental studies on single drop behavior in transitional and turbulent flows is still limited. As pointed out by Ashar et al. [3], the significant difficulties associated with measurement of single drop breakup is the limitation of optical access, problems with spatial resolution due to the small length scale of the drops, issues with temporal resolution due to the short lifetime of turbulent vortices and drop deformation/breakup time. Solsvik and Jakobsen [4] also indicated that manual data analyses of images to determine breakup time and the number of fragments is time consuming, while fully-automated tools for data analyses are difficult to design. Here we review several experimental studies that reveal details of drop breakup phenomena. We limit ourselves in this review to a single liquid drop breakup in another liquid under transitional to turbulent flow conditions. Some research groups [5,6] argue that drops (liquid-liquid systems) and bubbles (gas-liquid systems) have similar breakage mechanisms and that, therefore, their breakup mechanisms can be generalized as a breakage of fluid particles. However, as discussed by other groups, such as Becker et al. [7], the breakup mechanisms for drops and bubbles are fundamentally different. 
We support the latter point of view and distinguish between the breakup mechanisms of bubbles and drops.

Single drop behavior was investigated in flows generated by different devices, including stirred tanks, static mixers, rotor-stator mixers, as well as pipe flow and jets. The focus in each of the studies was to estimate the probability of drop breakup in a given flow condition, and to quantify the breakup time, as well as the number and size distribution of fragments after the breakup. Traditionally, many studies have been carried out in agitated tanks that have great practical relevance and - at the same time - complex and strongly inhomogeneous flow characteristics.

Konno et al. [8] investigated the formation of dispersion in a stirred tank using high-speed imaging. Low dispersed phase volume fractions in the range 0.001-0.002 were used to avoid coalescence between the drops. The size of the mother drops was in the range 0.26-1.0 mm. Two regions of drop breakup were outlined: inside and outside the impeller-disc edge. In the impeller-disc edge region, the dispersed drop was always elongated in the direction of the drop motion. However, outside the disc-edge region, the dispersed drop was elongated in random directions, independent of the drop's motion. Thus, no regularity in direction was observed. Based on this observation, the authors concluded that, outside the disc edge, the turbulent flow field is isotropic, while the region inside the edge is non-isotropic turbulent flow. Even though two different regions exist, the breakup characteristics obtained in these regions, such as breakup time and the number of daughter drops formed after breakup, were similar: the breakup time ranged from 1.4 to $6.9 \mathrm{~ms}$, and the average number of daughter drops was in the range 2.6-4.4. The latter indicates a low probability of binary breakup.

Maaß et al. [9] used high-speed imaging to study the drop breakup process in a stirred tank equipped with a Rushton turbine. The authors concluded that smaller mother drops produce fewer daughter drops. This means that a binary breakup has a high probability for smaller drops. For instance, a mother drop of $560 \mu \mathrm{m}$ in diameter showed a nearly $60 \%$ probability for binary breakage. Large drops $(\geq 2 \mathrm{~mm})$ most likely form satellites after breakage (ternary breakup). However, this conclusion might be not complete. A significant increase of energy input could decrease the probability of a binary breakup for small drops. 
Maaß et al. [10] performed experiments of single drop breakup in a breakage cell [11] and a lab-scale agitation vessel. The mother drop size varied between (500-3500) $\mu \mathrm{m}$. High-speed imaging was used to record the evolution of drop size distribution. Only the initial breakup events were analyzed meaning that the evolution of the mother drop was tracked up to the time instant when first (initial) disintegration occurred and the results of this initial breakup were recorded. The influence of the frame rate on the results, in terms of the drop count, was reported. As the frame rate of the camera increased, the number of binary breakages after the first breakage event increased, and vice versa for a decrease of the frame rate. That is, all ternary or higher breakage events are, most probably, a cascade of binary breakages. The maximum frame rate tested in this study was $1450 \mathrm{fps}$. To observe the outlined dependence of the drop count on the frame rate, the authors suggested a much higher time resolution.

Maaß and Kraume [12] used high-speed imaging to study the breakup rate of single drops in stirred tanks and single drop breakage cell under turbulent flow conditions. The mother drop size varied in the range of (0.54-3.2) mm. The authors provided a thorough discussion on drop breakup time as a function of mother drop diameter, liquid properties, and flow conditions. This time estimate is required for the models that describe the drop breakup process. However, there are several orders of magnitude in the variation of the breakup time estimates. The results of this experimental work provide important quantitative data on drop breakup time and promote awareness of what is widely assumed in the existing breakup models.

Solsvik and Jakobsen [4] studied a single drop breakup in a stirred tank equipped with Rushton turbine. The focus of the study was on the determination of the breakup time, the number of daughter drops, and the occurrence of equal and unequal breakup events. Unequal-sized breakage was more frequently observed than equal-sized breakup. Multiple breakup events were the most frequent outcomes in the experiments. The size of the mother drop, which was in the range of 0.6 to $4 \mathrm{~mm}$, affected the number of daughter drops produced in a breakup event. The authors outlined that the breakup time increased with the mother drop size and was in the approximate range of 10-100 ms. This study highlighted that inhomogeneity of the flow within the stirring device determines the probability of breakup: the drop is more likely to break in a region close to the radial impeller and significantly less likely to break in the bulk regions 
of the tank.

Galinat et al. [13] reported on a single drop breakup downstream of a restriction in a turbulent pipe flow. The high-speed camera was used to visualize drop breakup. The probability of drop breakup as a function of the flow conditions (flow Reynolds number) was correlated to the size of the mother drop. The authors reported that drops travelling in the core of the jet undergo quasi-symmetrical shape oscillations without breaking. Most of the breakup events occurred in the downstream flow at an axial distance greater than one pipe diameter. With the increase of the flow Reynolds number, multiple breakup events were observed.

Eastwood et al. [14] studied the breakup of immiscible liquids in turbulent water jets with high-speed imaging. Digital image-processing techniques were used to track the behavior of drops injected continuously on the centerline of the fully-developed region of a turbulent water jet. It was reported that the breakup process of dispersed drops with non-negligible density and viscosity at low Weber number disagrees with the classical Kolmogorov-Hinze theory for turbulent particle breakup: the dispersed drops stretch dramatically before fragmentation, even within locally isotropic regions of the flow. The calculated breakup frequency (inverse of the breakup time), scaled with the large-scale features of the turbulent flow, thus contradicting the classical theory premise that the structures responsible for the breakup are comparable in size to the diameter of the dispersed drops.

This review of experimental studies reveals that, to improve the reliability of drop breakup models, it is necessary to correlate the local hydrodynamic conditions of the flow around the drop with the outcome of the breakup event. The volume and time-averaged characteristics of turbulence are not sufficient to describe the turbulent breakage of drops $[15,16]$. Given significantly improved visualization techniques, it has become feasible to correlate a single drop breakup mechanism with local flow characteristics. For instance, the flow field in the custom-built rotor-stator mixer was comprehensively characterized by Håkansson et al. [17,18], using two-dimensional PIV measurements. The distribution of fragmenting stresses in the mixer was correlated with individual breakup events. Ashar et al. [3] also used high-speed camera imaging to study a single drop breakup in the same customized rotor-stator mixer. The authors then correlated the flow field simulated using computational fluid dynamics (CFD) 
with the experimental data on drop breakup. CFD simulations provide detailed information about the variability of stresses and the dissipation rate, connecting these data to the location and the cause of drop breakup. It was pointed out that, in the rotor-stator mixer, the drop breakup is controlled by the turbulent inertial stress acting on the drops.

These publications on drop breakup in rotor-stator equipment are essential steps towards a better understanding of the correlation between flow characteristics and breakup output. However, at the same time, the flow conditions in the rotor-stator mixer are specific to this device, wall/geometry-dominated, and challenging to control. Usually, experiments are performed either in laminar or turbulent flow conditions. Transitional flow is difficult to maintain experimentally or to simulate. However, in industrial applications, transitional flow occurs frequently. An effort to understand and generalize drop behavior in transitional flow conditions is important. In the present study, we experimentally investigate a single drop breakup in a more generic flow environment. Single silicon oil drops of known volume are injected into a deionized water jet with well-defined properies. The drop deformation and breakup are tracked using a high-speed imaging technique, while the jet flow is visualized using dye injection and quantified using two-dimensional particle image velocimetry (PIV). Our goal is to correlate the probability of drop breakup, breakup time and the size and number of fragments with the local flow conditions (velocity, stresses and energy dissipation rate).

The rest of the paper is organized as follows. The experimental facilities were first benchmarked by studying the buoyancy-driven motion of an oil drop in quiescent water. This part of the work is presented in Section 2.1. The description of the experimental setup of a jet flow, including dye visualization and 2D PIV, is given in Section 2.2-2.4. Section 3 contains the results and discussion. Section 4 concludes the paper.

\section{Experimental Setup}

\subsection{Experiments on a single drop rising in quiescent water}

The experiments of one drop rising in quiescent water were performed first to assess the accuracy of our experimental measurements and the reliability of our drop image processing. Fig. 1a shows the experimental setup. The size of the tank is $250 \mathrm{~mm}$ in length, $250 \mathrm{~mm}$ in width, and $600 \mathrm{~mm}$ in height. The tank was filled with deionized 
water up to a level of $500 \mathrm{~mm}$. The dispersed phase was dimethyl silicone oil (from Guangzhou Batai Chemical Co., LTD, China). We released the drops near the center of the bottom of the tank using a microinjection pump (Longer Precision Pump Co., Ltd, China) and different sharp needles ranged from 30-34 G (Birmingham Wire Gauge).

A high-speed camera GO-5000M-USB (JAI, Denmark) was used to capture the trajectory of the rising drop. The resolution of the images was $2560 \times 2048$ pixel $^{2}$, and the capture frequency was $50 \mathrm{fps}$. A light was installed opposite to the camera to obtain clear and distinct drop images. We adjusted the exposure time of the camera and found that the drop image was sufficiently clear when the exposure time was $1500 \mu \mathrm{s}$.

The electrical conductivity of the deionized water was $22.4 \mu \mathrm{S} / \mathrm{cm}(44.6 \mathrm{k} \Omega \cdot \mathrm{cm}$ in terms of specific resistance), which was measured by a DDSJ-308F conductivity meters (Shanghai INESA Scientific Instrument, China). This is water with a much lower specific resistance than the water used in the experiments of Bäumler et al. [19] which was highly purified deionized water with a specific resistance of $18.3 \mathrm{M} \Omega \cdot \mathrm{cm}$. The viscosities of the deionized water and the silicone oil as functions of temperature were measured by a HAAKE MARS40 rheometer (Thermo Fisher Scientific, USA). The relationship between dynamic viscosity (in $\mathrm{mPa} \cdot \mathrm{s}$ ) and temperature (in ${ }^{\circ} \mathrm{C}$ ) for the deionized water is $\mu_{\mathrm{C}}=-0.0234 T+1.468$ with $T$ in the range of 15 to $25{ }^{\circ} \mathrm{C}$. The relationship for the silicone oil is $\mu_{\mathrm{D}}=-0.0509 T+3.4786$ in the same temperature range. The interfacial tension between the deionized water and the silicone oil was measured by a contact angle measuring instrument OCA50AF (Dataphysics, Germany). The interfacial tension (in $\mathrm{mN} / \mathrm{m}$ ) as a function of temperature (in ${ }^{\circ} \mathrm{C}$ ) is $\gamma=-0.415 T+44.068$ when $T$ ranges from 15 to $25{ }^{\circ} \mathrm{C}$. The experimental temperature was controlled at $20.0 \pm 1.0{ }^{\circ} \mathrm{C}$ so that $\mu_{\mathrm{C}}=1.000 \pm 0.023 \mathrm{mPa} \cdot \mathrm{s}$, $\mu_{\mathrm{D}}=2.461 \pm 0.050 \mathrm{mPa} \cdot \mathrm{s}$, and $\gamma=35.77 \pm 0.42 \mathrm{mN} / \mathrm{m}$. The maximum viscosity variation caused by the temperature fluctuation $\left( \pm 1.0^{\circ} \mathrm{C}\right)$ is about $3 \%$. The densities of the deionized water and the silicone oil are $998 \mathrm{~kg} / \mathrm{m}^{3}$ and $837 \mathrm{~kg} / \mathrm{m}^{3}$, respectively, at $20.0{ }^{\circ} \mathrm{C}$. The error of density measurements is less than $0.1 \%$. The physical properties of the silicone/water system are summarized in Table 1.

As will be presented below, the experiments show - in terms of drop rise velocities - good agreement with the correlation from literature related to contaminated systems [20]. In order to further check the characteristics of our 
flow system we also replicated the experiments by Bäumler et al. [19] on toluene/water systems with special attention to the properties of water we used. In the experiments of drop rising in quiescent water (silicone oil/water systems and toluene/water systems), the diameter of drops was the only variable. The drop Reynolds number ranged from 75 to 566 .

In these benchmark experiments the temperature was controlled at $25.0 \pm 1.0{ }^{\circ} \mathrm{C}$. Toluene with a purity of $99.5 \%$ provided by Beijing Chemical Works (China) was used as the dispersed phase. The same deionized water as in the silicone oil/water system was used as the continuous phase. Physical parameters of the toluene/water system were tested with the same methods as used for the silicone oil/water system, see Table 1 . Table 1 also lists the physical properties of the toluene/water system as used by Bäumler et al. [19].

\subsection{Drop breakup in jet flow experiments}

The experimental setup for the drop breakup in a jet is shown in Fig. 1b. In order to generate a stable and controllable jet flow field, we carefully designed a flow system, including a diaphragm pump, a damper, a buffer tank, a pressure gauge, a back pressure valve, and two needle valves. As the diaphragm pump is a positive displacement pump, a damper and a buffer tank were used to eliminate the periodic fluctuations in the flow rate. The liquid pressure in the pipeline indicated in red in Fig. 1b was approximately $2.5 \mathrm{~atm}$. The back pressure valve was used for keeping the liquid pressure, as well as for ensuring safety in the experiments. The two needle valves were used for adjusting the flow rate. The reading of the pressure gauge reflected the stability of the flow rate. Its fluctuations were less than $1 \%$ of the working pressure. We conclude that the flow rate of the jet was sufficiently stable. In the experiments of drop breakup, the jet velocity was the only variable. The jet Reynolds ranged from 1283 to 1610 .

The tank was filled up to a height of $180 \mathrm{~mm}$ with deionized water. A lid was placed on the free surface of the deionized water to prevent liquid surface fluctuation. The cross section of the jet pipe is a square with side length of $5.20 \mathrm{~mm}$. The origin of the Cartesian coordinate system is located at the cross-section center of the outlet of the jet pipe (see Fig. 1b). The length of the jet pipe is $280 \mathrm{~mm}$ to ensure that the flow inside the pipe fully develops. The Reynolds number of the jet flow is defined as $R e=\rho_{\mathrm{C}} \bar{u} d_{\mathrm{a}} / \mu_{C}$, where $\mu_{\mathrm{C}}$ denotes the viscosity of the deionized 
water, $\rho_{\mathrm{C}}$ is the density of the deionized water, $\bar{u}$ is the average velocity of the jet flow, and $d_{\mathrm{a}}$ is the hydraulic diameter of the jet which is calculated as $d_{\mathrm{a}}=4 A / C=l$ with $A, C$ and $l$ the cross sectional area, perimeter and side length of the jet pipe, respectively. The vertical distance from the center of the jet pipe to the tank bottom (in $y$ direction) is $70 \mathrm{~mm}$.

Needles with the inner diameter of $1.3 \mathrm{~mm}$ and the outer diameter of $1.6 \mathrm{~mm}$ and flat outlet shape were used to generate drops. The center lines of the jet pipe and the needle are in the same $x-y$ plane (with $z=0$ ). The horizontal distance in $x$ direction between the two outlet centers of the needle and the jet pipe is $19.48 \mathrm{~mm}$, and the vertical distance in $y$ direction between the two centers is $10.84 \mathrm{~mm}$ (see Fig. 1b).

The continuous phase was deionized water, and the dispersed phases was silicone oil as mentioned in Section 2.1. The temperature was controlled at $20.0 \pm 1.0{ }^{\circ} \mathrm{C}$ as well. The camera, image resolution, capture frequency, exposure time, and light arrangement were the same as those in the experiments in Section 2.1.

\subsection{Drop detection}

Accurately detecting the edges of the drops and then calculating the drop sizes are of vital importance in our two sets of experiments. The Canny edge detection method [21] was used for identifying the edge of the drops in the camera frames. Fig. 2 shows three raw images of drops in the jet flow experiments and the corresponding detection results. The drops close to the needle in both sets of experiments could be considered as axisymmetric along a center line in $y$-direction because the continuous liquid is at rest in the experiments of Section 2.1 and the influence of the jet on the drops near the needle is negligible in the experiments of Section 2.2. The volume of a drop was calculated as $V_{\mathrm{D}}=\int_{y_{1}}^{y_{2}} \pi R(y)^{2} d y$ with $y_{1}$ and $y_{2}$, the highest and lowest positions of the drop, respectively, and $R$ the radius of the drop as a function of $y$. The difference in the calculated volumes of a drop near the needle is less than $\pm 0.2 \%$, for example, the volume of the drop as calculated from the sequence of images Figs. 2d, 2e, and $2 \mathrm{f}$ is $97.60 \mu \mathrm{l}, 97.62 \mu \mathrm{l}$, and $97.36 \mu \mathrm{l}$, respectively. The centroid of a drop was calculated as $\bar{x}=\left(\int_{y_{1}}^{y_{2}} \int_{x_{1}(y)}^{x_{2}(y)} x d x d y\right) /\left(\int_{y_{1}}^{y_{2}} \int_{x_{1}(y)}^{x_{2}(y)} d x d y\right)$ and $\bar{y}=\left(\int_{y_{1}}^{y_{2}} \int_{x_{1}(y)}^{x_{2}(y)} y d x d y\right) /\left(\int_{y_{1}}^{y_{2}} \int_{x_{1}(y)}^{x_{2}(y)} d x d y\right)$ with $x_{1}(y)$ and $x_{2}(y)$ the left and right boundaries of the drop respectively, as shown in Fig. 2 d. 
The drop diameter in the experiments of rising drops ranges from 2.16 to $3.83 \mathrm{~mm}$ for silicone oil drops and from 1.50 to $4.47 \mathrm{~mm}$ for toluene drops. In the second set of experiments (drops interacting with jet flow), we released 425 drops in total. The average volume of the drops is $V_{\mathrm{av}}=97.9 \pm 1.0 \mu \mathrm{L}$, and then the equivalent diameter $\left(d_{\mathrm{D}}\right)$ is $5.72 \pm 0.03 \mathrm{~mm}$.

\subsection{Single-phase jet flow visualization and 2D PIV}

Two visualization methods were used to investigate the jet flow. First, we added ink to the flow near the inlet of the jet pipe, and qualitatively showed the characteristics of the jet flow at different Reynolds numbers. Second, we used the 2D PIV technique to quantitatively measure the jet flow velocity field. The results are discussed in Section 3.3.

The 2D PIV system (TSI USA) consists of a $532 \mathrm{~nm} 200 \mathrm{~mJ}$ Nd:YAG dual pulse laser (model No: Vlite-200, Beamtech, China), spherical and cylindrical lenses which transform a laser beam into a laser sheet with thickness of 1 $\mathrm{mm}$ at the measurement plane, a 4008x2672 pixels charge coupled device (CCD) camera (model No: PowerView Plus 11M, TSI, USA), a synchronizer (model No: Laser Pulse 610035, TSI, USA), and Insight 3G software. Hollow glass beads (TSI, USA) with diameter of 8-12 $\mu \mathrm{m}$ were used as tracer particles.

In the PIV experiments, the time interval $\Delta t$ between the two images in a pair was determined by examining the displacements of illuminated particles and was optimized as $\Delta t=400 \mu$ s to ensure that the maximum in-plane and out-of-plane displacements of seeding particles were less than one-quarter of the interrogation spot size and the thickness of the laser sheet. To resolve the flow field in the region of drop breakup, a rectangular area of approximately $90 \mathrm{~mm}$ x $60 \mathrm{~mm}$ was captured, as marked by the red frame in Fig. 1b. The size of each of the interrogation windows was $48 \times 48$ pixel $^{2}$ with $50 \%$ overlap between the windows. The resolution of the PIV images was $0.0227 \mathrm{~mm} / \mathrm{pixel}$, and then the velocity vector resolution was $0.55 \mathrm{~mm}$. The PIV capture frequency was $1 \mathrm{~Hz}$. Six hundred PIV realizations were measured at each Reynolds number and then were analyzed to obtain average flow field data. Erroneous instantaneous vectors were deleted by using the median filtering method [22]. The missing vectors were filled using a linear interpolation method [23]. 
The Kolmogorov length scale of the jet flow was estimated as $\eta=d_{\mathrm{a}} R e^{-3 / 4}$ with $d_{\mathrm{a}}$ the hydraulic diameter of the jet pipe. It should be noted that this is a coarse estimate for $\eta$ since it is based on fully developed turbulence which we do not have. With $R e$ in the range of 1280 to $1610, \eta$ was $0.020-0.024 \mathrm{~mm}$. As a result, the Kolmogorov length scale is much smaller than the PIV resolution. The dissipation rate of turbulent kinetic energy (TKE) $\varepsilon$ plays an important role in drop breakup models [24,25]. Given the limited resolution of the PIV experiments, we apply a large eddy method [26] to estimate $\varepsilon$ from the PIV data:

$$
\langle\varepsilon\rangle=2^{3 / 2} C_{S}^{2} \Delta^{2}\left\langle S^{3}\right\rangle
$$

where $C_{\mathrm{S}}$ is the Smagorinsky constant which depends on the degree of windows overlap [27], $\Delta$ is the size of the interrogation window, and $S$ is $\left(\sum_{\mathrm{i}, \mathrm{j}} S_{\mathrm{ij}} S_{\mathrm{ij}}\right)^{1 / 2}$ with $S_{\mathrm{ij}}=\frac{1}{2}\left(\frac{\partial u_{\mathrm{i}}}{\partial x_{\mathrm{j}}}+\frac{\partial u_{\mathrm{j}}}{\partial x_{\mathrm{i}}}\right)$ the strain rate tensor associated to the velocity field resolved by the PIV. In line with Bertens et al. [27], we set $C_{\mathrm{S}}=0.19$ for $50 \%$ overlap of interrogation windows. The term $\left\langle S^{3}\right\rangle$ in Eq. 1 is assumed to be equal to $\left\langle S^{2}\right\rangle^{3 / 2}$ [28]. Since 2D PIV is not able to measure all components of the strain rate tensor, there is a need for isotropy assumptions $[29,30]$ to estimate the missing terms in the tensor. Then $\left\langle S^{2}\right\rangle$ is calculated as [27]

$$
\left\langle S^{2}\right\rangle=\frac{3}{2}\left(\left\langle\left(\frac{\partial u_{1}}{\partial x_{2}}\right)^{2}\right\rangle+\left\langle\left(\frac{\partial u_{2}}{\partial x_{1}}\right)^{2}\right\rangle\right)+\frac{3}{4}\left(\left\langle\left(\frac{\partial u_{1}}{\partial x_{1}}\right)^{2}\right\rangle+\left\langle\left(\frac{\partial u_{2}}{\partial x_{2}}\right)^{2}\right\rangle\right)
$$

In a Reynolds decomposition, the instantaneous velocity field is viewed as a superposition of the mean velocity and the fluctuating velocity: $u_{\mathrm{i}}=\bar{u}_{\mathrm{i}}+u_{\mathrm{i}}{ }^{\prime}$. In this paper, we therefore distinguish between the total energy dissipation rate and the dissipation rates contained in the average flow and in the fluctuating flow respectively by using the respective velocity contributions when evaluating $S_{\mathrm{ij}}$. As a result, $\left\langle S^{2}\right\rangle_{\text {total }}=\left\langle S^{2}\right\rangle_{\mathrm{av}}+\left\langle S^{2}\right\rangle_{\text {fluct }}$ and thus - given that $\left.\left\langle S^{3}\right\rangle \approx\left\langle S^{2}\right\rangle^{3 / 2}-\langle\varepsilon\rangle_{\text {total }}\right\rangle\langle\varepsilon\rangle_{\text {av }}+\langle\varepsilon\rangle_{\text {fluct }}$.

With the estimated $\varepsilon$, the shear rate $r^{\prime}$ was estimated as $r^{\prime}=(\varepsilon / v)^{1 / 2}$ with $v$ the kinematic viscosity of the continuous liquid (water) [31].

\section{Results and Discussion}

\subsection{Drop rising in quiescent water}

Series of images of oil drops rising in quiescent ambient water are shown in Fig. 3. We determine the vertical 
velocities of the drops by the vertical displacement of the centroid over the time interval between two subsequent images. The terminal velocities for different drop diameters are shown in Table 2 and 3 . All the drops are to a very good approximation spherical. When the Reynolds number based on the drop diameter, rise velocity, and the viscosity of the continuous phase fluid $\left(R e_{\mathrm{D}}=\rho_{\mathrm{C}} d_{\mathrm{D}} u_{\mathrm{D}} / \mu_{\mathrm{C}}\right)$ is larger than 200 (Fig. $3 \mathrm{f}, R e_{\mathrm{D}}=214$; Fig. $3 \mathrm{~g}, R e_{\mathrm{D}}=$ 241), the path of the drop starts to oscillate slightly. Volkov et al. [32, 33] reported the deformation cycles (spheroid - ellipsoid - spheroid) during their study on the features of deformation of water droplets (3-6 mm) in gaseous medium $(275-1100 \mathrm{~K})$ with velocities from $0.5 \mathrm{~m} / \mathrm{s}$ to $5 \mathrm{~m} / \mathrm{s}$.

In order to validate our experimental results on drop rise velocities, we relate them to the well-known flow chart for rising drops and bubbles due to Grace et al. [20]. That chart shows $R e_{\mathrm{D}}$ as a function of the Morton number $\left(M=g \mu_{\mathrm{C}}^{4} \Delta \rho / \rho_{\mathrm{C}}^{2} \gamma^{3}\right)$ and Eötvös number $\left(E o=\Delta \rho g d_{\mathrm{D}}^{2} / \gamma\right)$. For all silicone oil drops in water, $\log _{10}(M)=-10.45$. As for the toluene drops in water, $\log _{10}(M)=-10.59$. The experimental data on drop size and rise velocity in this work and from Bäumler et al. [19] were converted to $E o$ and $R e$ and have been plotted in the flow chart (see Fig. 4). For the silicone oil/water system, as well as for the toluene/water system, our experimental data are in good agreement with the corresponding curves from contaminated systems $\left(\log _{10}(M)=-10.45\right.$ and -10.59 , respectively). The main reason for considering our systems as contaminated is that the specific resistance of the water used in our work is significantly lower as compared to highly purified deionized water as used by Bäumler et al. [19], see Section 2.1. This then explains the difference between the rise velocities of toluene drops through water as measured by Baumler et al. [19] and by us. Uncertainty analysis for drop rising in quiescent water was given in the Appendix (a). The so obtained levels of uncertainly allow for a meaningful comparison with drop rise data.

\subsection{Drop deformation and breakup in jet flow}

In the experimental set-up as shown Fig. 1b, the flow rate is adjusted through the needle valve. Once the valve was adjusted and the pressure gauge was stable, we calibrated the flow rate by weighing the outflow over a precise time interval. Uncertainty analysis for the jet Reynolds number was given in the Appendix (b). The so obtained levels of uncertainly allow for a meaningful characterization of the jet flow. It took approximately $9 \mathrm{~s}$ to generate and 
release a silicone oil drop. Its trajectory, deformation and possible breakup in the jet flow was then filmed. The total number of drops in this set of experiments was 425 .

Table 4 summarizes the drop-jet interaction results. As we will see in the next Section, the jet goes through a transition from laminar to transitional in this Re range. Initially, the drop breakup probability sharply increases with increasing $R e$. Where for $R e=1283$ none of the drops is broken when passing through the jet flow (although they are significantly deformed by the jet), an increase to approximately $R e=1313$ (an increase by only $2.5 \%$ ) leads to an almost $100 \%$ breakup yield. This yield remains constant up to $R e=1501$. Beyond this value the breakup probability decreases and - at the same time - some daughter drops break for a second time (breakup cascade [34]). The reduction of breakup probability at higher $R e$ is due to the fact that the drops are not able to penetrate the jet when it gets stronger; they are deflected horizontally.

Fig. 5 shows the trends identified in Table 4 graphically. Sample images capturing breakup are given in Fig. 6 and 7. In Fig. 6 (frames $e, f$, and $g$ ) it can be clearly seen how the drop is hit by the jet, gets dented, and as a result breaks into two fragments in frame $h$. When the Reynolds number is increased, from $R e=1333$ in Fig. 6, to 1536 in Fig. 7, the drop breaks further downstream of the jet because the drop is deflected horizontally by the jet and, ultimately breaks in three fragments.

Fig. 8 shows the average volume of the upper daughter drop as a function of $R e$. The volume of upper daughter drop is larger than the lower one if $R e$ is low, and decreases as $R e$ goes up. An equal split between the two daughter drops is achieved when $R e$ is approximately 1480 . The interpretation of these results will be discussed after we have presented the jet flow field measurements.

\subsection{Jet flow field}

The dye visualizations of the jet in Fig. 9 show a transition from laminar to transitional. Where the jet is stable and laminar over at least 5 hydraulic diameters downstream of the pipe exit for $R e=1293$, the jet at $R e=1531$ turns transitional within two diameters downstream the pipe exit. With the location of the needle from which droplets emanate indicated in Fig. 9, it is clear that droplets experience very different flow fields at different jet Reynolds 
numbers. The sample PIV results in Fig. 10 confirm what is observed in the dye visualizations and also show the transition.

Figs. $11 \mathrm{a}$ and $11 \mathrm{~b}$ show that at $R e=1317$ the dissipation rate of TKE in the average flow is much higher than in the fluctuating flow. In Fig. 11 we also verify that the dissipation directly calculated from the PIV velocity fields (i.e. without performing a $R e$ decomposition) is slightly larger than the sum of dissipation contained in the average flow and in the fluctuations. The dissipation rate in the fluctuating flow increases with the increase of $R e$, see Fig. 12.

\subsection{Mechanism of drop breakup}

Since the drops hinder the optical access required for PIV, given the differences in refractive between the two liquids (silicon oil and water), we are not able to perform drop breakup visualization and PIV experiment simultaneously. It is realized, however, that drop deformation and breakup are governed by capillary and/or Weber numbers, average contours of which can be determined - given drop size and surface tension - based on the PIV results:

$$
\begin{aligned}
& C a=\mu_{\mathrm{C}} d_{\mathrm{D}} r^{\prime} / 2 \gamma \\
& W e=\rho_{\mathrm{C}} \bar{u}^{2} d_{\mathrm{D}} / \gamma
\end{aligned}
$$

where $\mu_{\mathrm{C}}$ is the dynamic viscosity of deionized water, $\rho_{\mathrm{C}}$ is the density of deionized water, $d_{\mathrm{D}}$ is the diameter of drop, $\gamma$ is the interfacial tension, $r^{\prime}$ is the shear rate calculated by $r^{\prime}=(\varepsilon / v)^{1 / 2}$, and $\bar{u}$ is the average velocity of the flow.

Such contours are shown in Figs. 13 and 14, for capillary and Weber number respectively and are combined with representative drop visualizations where the Reynolds numbers of drop visualization experiment $\left(R e_{1}\right)$ and PIV experiment $\left(R e_{2}\right)$ are closely matched. At the lowest Reynolds numbers considered (Re $\approx 1285$; Panel (a) in Fig. 13 and 14) the sample drop deforms but does not break. The drop deformation is mostly an inertial effect: the location of a dent in the drop coincides with peak levels in the Weber number; at the same location the capillary number has a local minimum. The other three panels in Figs. 13 and 14 deal with drop break up, with Panel (d) showing a breakup cascade event as well. In all cases, drop deformation starts on the center line of the jet indicating - see above - 
inertial effect. The actual breakup, however, seems to occur as a result of the neck formed initially from inertial denting the drop is sheared off in the high shear (i.e. high $\mathrm{Ca}$ ) region. Thus, we consider that breakage is due to combined inertial and viscous (shear) effects, which is slightly different from the four main breakup mechanisms in turbulent dispersions: (1) turbulent fluctuation and eddy-drop collision; (2) viscous shear stress; (3) shearing-off process; (4) interfacial instability [35].

Fig. 8 shows that, when breakup occurs, the volume of the upper fragment gets relatively smaller when the jet Reynolds number increases. Given that the breakup process is initiated through inertial effects that are strongest on the centerline of the jet and are increasing with increasing jet Reynolds number, drops - while rising into the jet will get deformed earlier so that the upper fragments get sheared off sooner when the jet Reynolds number increases.

The results in Figs. 13 and 14 provide an order of magnitude estimate for critical capillary and Weber numbers for drop breakup in our jet flow system: critical $\mathrm{Ca}$ is of the order of 0.2 , critical We is of the order of 30 .

\section{Conclusions}

In this study, we constructed a well-defined liquid jet flows and investigated the characteristics of drop breakup in the jet. Silicone oil and deionized water were chosen as the dispersed phase and continuous phase, respectively. The process of drop breakup was captured using quantitative visualization. To assess the accuracy of the experimental system and the reliability of the image processing methods used, we performed benchmark experiments with silicone oil/water and toluene/water systems. The latter to investigate the impact of the water properties (specifically conductivity) on the behavior of the droplet phase. Taking the water conductivity into account, the results of the benchmark experiments are in good agreement with the seminal results collected by Grace et al. [20].

With the thus verified silicone oil/deionized water system, we visualized drop breakup in laminar and transitional jet flows and quantified the results in terms of breakup probability, breakup time, and number and volume distributions of daughter drops. When the jet Reynolds number goes beyond a critical value of approximately 1310 , the breakup percentage becomes near 100\%. Breakup cascade occurs at jet Reynolds numbers in the range of 1500 to 1610. Drop breakup patterns and daughter drop volumes are thus closely related to the jet Reynolds number. 
This led to an experimental study of the (single-phase) jet flow characteristics as a function of the Reynolds number. The results clearly show the change of the flow field from laminar to transitional. Combining the drop breakup results and single-phase 2D PIV data, we were able to estimate critical conditions for the drop breakup in this jet flow: critical capillary and Weber numbers calculated based on the average flow field are of the order of 0.2 and 30, respectively.

Where in the current study drop break-up and (single-phase) jet flow were investigated separately, future research will attempt to study these simultaneously so that the two-way coupling between the two liquids is visualized. For this, optical access is a critical issue with the drops likely scattering the laser light required to do PIV. This hurdle can be overcome by (near) refractive index matching of the liquids involved [36]. It will also be very relevant to perform detailed, interface-resolving direct numerical simulations of the systems studied experimentally in this paper.

\section{Acknowledgement}

The authors gratefully acknowledge the financial support from the National Key R\&D Program of China (2017YFB0306701), National Natural Science Foundation of China (No.21676007), the Fundamental Research Funds for the Central Universities (XK1802-1), and Scientific Research and Technology Development Projects of China National Petroleum Corporation (No. 2016B-2605). 


\section{Nomenclature}

\begin{tabular}{|c|c|}
\hline \multicolumn{2}{|c|}{ Abbreviations } \\
\hline CCD & charge coupled device \\
\hline CFD & computational fluid dynamics \\
\hline DSD & drop size distribution \\
\hline PIV & particle image velocimetry \\
\hline TKE & turbulent kinetic energy \\
\hline \multicolumn{2}{|c|}{ dimensionless numbers } \\
\hline $\mathrm{Ca}$ & Capillary number \\
\hline Eo & Eötvös number \\
\hline$M$ & Morton number \\
\hline$R e$ & Reynolds number of the jet flow \\
\hline$R e_{\mathrm{D}}$ & Reynolds number of drop \\
\hline We & Weber number \\
\hline \multicolumn{2}{|c|}{ Latin symbols } \\
\hline$A$ & sectional area of jet pipe, $\mathrm{m}^{2}$ \\
\hline$C$ & perimeter of jet pipe, $m$ \\
\hline$C_{\mathrm{S}}$ & Smagorinsky-Lilly constant, - \\
\hline$d_{\mathrm{a}}$ & hydraulic diameter, $\mathrm{m}$ \\
\hline$d_{\mathrm{D}}$ & equivalent diameter of drop, $\mathrm{m}$ \\
\hline$l$ & side length of jet pipe, $\mathrm{m}$ \\
\hline$r^{\prime}$ & shear rate, $\mathrm{s}^{-1}$ \\
\hline$S$ & strain rate tensor, $\mathrm{s}^{-1}$ \\
\hline$T$ & temperature, ${ }^{\circ} \mathrm{C}$ \\
\hline$u_{\mathrm{D}}$ & terminal velocity of dispersed phase, $\mathrm{m} / \mathrm{s}$ \\
\hline$u_{\mathrm{i}}$ & instantaneous velocity of the flow, $\mathrm{m} / \mathrm{s}$ \\
\hline $\bar{u}$ & average velocity of the flow, $\mathrm{m} / \mathrm{s}$ \\
\hline$u_{\mathrm{i}}^{\prime}$ & fluctuating velocity of the flow, $\mathrm{m} / \mathrm{s}$ \\
\hline
\end{tabular}




\begin{tabular}{|l|l|}
\hline$V_{\mathrm{av}}$ & average volume of drop, $\mathrm{m}^{3}$ \\
\hline$V_{\mathrm{D}}$ & volume of drop, $\mathrm{m}^{3}$ \\
\hline$V_{1, \text { av }}$ & average volume of the upper daughter drop after breakup, $\mathrm{m}^{3}$ \\
\hline & \\
\hline Greek symbols \\
\hline$\gamma$ & interfacial tension, $\mathrm{N} / \mathrm{m}$ \\
\hline$\Delta$ & size of the interrogation window, $\mathrm{m}$ \\
\hline$\Delta \rho$ & density difference, $\mathrm{kg} / \mathrm{m}^{3}$ \\
\hline$\Delta t$ & time interval, $\mathrm{s}$ \\
\hline$\varepsilon$ & dissipation rate of turbulent kinetic energy, $\mathrm{m}^{2} \mathrm{~s}^{-3}$ \\
\hline$\langle\varepsilon\rangle_{\text {total }}$ & total dissipation rate in the instantaneous flow, $\mathrm{m}^{2} \mathrm{~s}^{-3}$ \\
\hline$\langle\varepsilon\rangle_{\text {av }}$ & dissipation rate in the average flow, $\mathrm{m}^{2} \mathrm{~s}^{-3}$ \\
\hline$\langle\varepsilon\rangle_{\text {fluct }}$ & dissipation rate in the fluctuating flow, $\mathrm{m}^{2} \mathrm{~s}^{-3}$ \\
\hline$\eta$ & Kolmogorov scale, $\mathrm{m}$ \\
\hline$\mu_{\mathrm{C}}$ & viscosity of continuous phase, Pa $\mathrm{s}$ \\
\hline$\mu_{\mathrm{D}}$ & viscosity of dispersed phase, Pa $\cdot \mathrm{s}$ \\
\hline$\mu_{\mathrm{w}}$ & viscosity of deionized water, Pa $\cdot \mathrm{s}$ \\
\hline$v$ & kinematic viscosity of continuous phase, $\mathrm{m}^{2} / \mathrm{s}$ \\
\hline$\rho_{\mathrm{C}}$ & density of continuous phase, $\mathrm{kg} / \mathrm{m}^{3}$ \\
\hline$\rho_{\mathrm{D}}$ & density of dispersed phase, $\mathrm{kg} / \mathrm{m}^{3}$ \\
\hline
\end{tabular}




\section{Appendix}

\section{(a) Uncertainty analysis for drop rising in quiescent water}

Uncertainty of the physical parameters due to systematic error: $\rho_{\mathrm{C}}, 0.100 \% ; \rho_{\mathrm{D}}, 0.100 \%$, estimated from the systematic error of balance $(3000 \pm 0.01 \mathrm{~g})$ and volumetric flask $(1000 \pm 0.8 \mathrm{ml}) . \mu, 2.07 \% ; \gamma, 2.37 \%$, estimated from the maximum deviation between actual and theoretical values.

Uncertainty of the physical parameters due to temperature $\left(1{ }^{\circ} \mathrm{C}\right): \mu_{\mathrm{C}}, 2.30 \% ; \mu_{\mathrm{D}}, 2.04 \% ; \gamma, 1.18 \%$.

Thus, the uncertainty of the physical parameters: $\rho_{\mathrm{C}}, 0.100 \% ; \rho_{\mathrm{D}}, 0.100 \% ; \mu_{\mathrm{C}},\left(2.07 \%^{2}+2.30 \%^{2}\right)^{0.5}=3.10 \%$; $\mu_{\mathrm{D}},\left(2.07 \%^{2}+2.04 \%^{2}\right)^{0.5}=2.91 \% ; \gamma,\left(2.37 \%^{2}+1.18 \%^{2}\right)^{0.5}=2.65 \%$.

The uncertainty of $d_{\mathrm{D}}$ and $u_{\mathrm{D}}$ are estimated as follows:

In our drop rising up experiment, the resolution is $0.01525 \mathrm{~mm} /$ pixel. The diameters of drop in Tables 2 and 3 are between 1.50 and $4.47 \mathrm{~mm}$. The maximum uncertainty of $d_{\mathrm{D}}$ (for smallest drop) is $0.01525 / 1.50=1.02 \%$. The terminal velocities $u_{\mathrm{D}}$ of the drops are between $44.7 \mathrm{~mm} / \mathrm{s}$ and $113.2 \mathrm{~mm} / \mathrm{s}$. As the central difference method was used to calculate the velocity, the uncertainty of $u_{\mathrm{D}}$ (assuming there is no significant error in $\Delta t$ ) is $2 \times 0.01525 /(44.7 \times 2 \times \Delta t)=2 \times 0.01525 /(44.7 \times 0.04)=1.71 \%$.

The relative uncertainty [37] in the $M=\frac{g \mu_{\mathrm{C}}^{4} \Delta \rho}{\rho_{\mathrm{C}}^{2} \gamma^{3}}:\left((3.10 \% \times 4)^{2}+(0.100 \%)^{2}+(0.100 \% \times 2)^{2}+(2.65 \% \times 3)^{2}\right)^{0.5}=$ $14.8 \%$.

$\log _{10}(M): \log _{10}\left(3.55 \times 10^{-11}\right)=-10.45 ; \log _{10}\left(1.148 \times 3.55 \times 10^{-11}\right)=-10.39 ; \log _{10}\left(0.852 \times 3.55 \times 10^{-11}\right)=-10.52$.

The relative uncertainty in the $R e_{\mathrm{D}}=\frac{\rho_{\mathrm{C}} d_{\mathrm{D}} u_{\mathrm{D}}}{\mu_{\mathrm{C}}}:\left((0.100 \%)^{2}+(1.02 \%)^{2}+(1.71 \%)^{2}+(3.10 \%)^{2}\right)^{0.5}=3.69 \%$.

The relative uncertainty in the $E o=\frac{\Delta \rho g d_{\mathrm{D}}^{2}}{\gamma}:\left((0.100 \%)^{2}+(1.02 \% \times 2)^{2}+(2.65 \%)^{2}\right)^{0.5}=3.35 \%$.

\section{(b) Uncertainty analysis for drop breakup in the jet flow}

The uncertainty of jet pipe $d_{\mathrm{a}}$ is $0.01 / 5.20=0.193 \%$. Uncertainty of jet velocity: $1.00 \%$ (The reading of the pressure gauge reflected the stability of the flow rate. Its fluctuations were less than $1 \%$ of the working pressure).

The relative uncertainty in the jet Re number: $\left((0.100 \%)^{2}+(1.00 \%)^{2}+(0.193 \%)^{2}+\left((3.10 \%)^{2}\right)^{0.5}=3.27 \%\right.$.

The so obtained levels of uncertainly allow for (a) meaningful comparison with drop rise data and (b) for a 
meaningful characterization of the jet flow.

\section{References}

[1] T.F. Tadros, Emulsion formation, Stability, and Rheology, in: T.F. Tadros (Ed.) Emulsion formation and Stability, Wiley-VCH Verlag GmbH \& Co. KGaA, Weinheim, Germany, 2013, pp. 1-75.

[2] M.M. Ribeiro, P.F. Regueiras, M.M.L. Guimarães, C.M.N. Madureira, J.J.C. Cruz_Pinto, Optimization of breakage and coalescence model parameters in a steady-state batch agitated dispersion, Industrial \& Engineering Chemistry Research 50 (2011) 2182-2191.

[3] M. Ashar, D. Arlov, F. Carlsson, F. Innings, R. Andersson, Single droplet breakup in a rotor-stator mixer, Chemical Engineering Science 181 (2018) 186-198.

[4] J. Solsvik, H.A. Jakobsen, Single drop breakup experiments in stirred liquid-liquid tank, Chemical Engineering Science 131 (2015) 219-234.

[5] R.P. Hesketh, A.W. Etchells, T.W.F. Russell, Experimental observations of bubble breakage in turbulent flow, Industrial \& Engineering Chemistry Research 30 (1991) 835-841.

[6] C. Xing, T. Wang, K. Guo, J. Wang, A unified theoretical model for breakup of bubbles and droplets in turbulent flows, AIChE Journal 61 (2015) 1391-1403.

[7] P.J. Becker, F. Puel, R. Henry, N. Sheibat-Othman, Investigation of discrete population balance models and breakage kernels for dilute emulsification systems, Industrial \& Engineering Chemistry Research 50 (2011) 11358-11374.

[8] M. Konno, M. Aoki, M. Saito, Scale effect on breakup process in liquid-liquid agitated tanks, Journal of Chemical Engineering of Japan 16 (1983) 312-319.

[9] S. Maaß, A. Gäbler, A. Zaccone, A.R. Paschedag, M. Kraume, Experimental investigations and modelling of breakage phenomena in stirred liquid/liquid systems, Chemical Engineering Research and Design 85 (2007) 703-709.

[10] S. Maaß, S. Buscher, S. Hermann, M. Kraume, Analysis of particle strain in stirred bioreactors by drop breakage investigations, Journal of Biotechnology 6 (2011) 979-992.

[11] S. Maaß, S. Wollny, R. Sperling, M. Kraume, Numerical and experimental analysis of particle strain and breakage in turbulent dispersions, Chemical Engineering Research and Design 87 (2009) 565-572.

[12] S. Maaß, M. Kraume, Determination of breakage rates using single drop experiments, Chemical Engineering Science 70 (2012) 146-164.

[13] S. Galinat, O. Masbernat, P. Guiraud, C. Dalmazzone, C. Noïk, Drop break-up in turbulent pipe flow downstream of a restriction, Chemical Engineering Science 60 (2005) 6511-6528.

[14] C.D. Eastwood, L. Armi, J.C. Lasheras, The breakup of immiscible fluids in turbulent flows, Journal of Fluid Mechanics 502 (2004) 309-333.

[15] F. Ghasempour, R. Andersson, B. Andersson, D.J. Bergstrom, Number density of turbulent vortices in the entire energy spectrum, AIChE Journal 60 (2014) 3989-3995.

[16] F. Ghasempour, R. Andersson, B. Andersson, Identification and characterization of three-dimensional turbulent flow structures, AIChE Journal 62 (2016) 1265-1277.

[17] A. Håkansson, H.H. Mortensen, R. Andersson, F. Innings, Experimental investigations of turbulent fragmenting stresses in a rotor-stator mixer. Part 1. Estimation of turbulent stresses and comparison to breakup visualizations, Chemical Engineering Science 171 (2017) 625-637.

[18] A. Håkansson, R. Andersson, H.-H. Mortensen, F. Innings, Experimental investigations of turbulent fragmenting stresses in a rotor-stator mixer. Part 2. Probability distributions of instantaneous stresses, Chemical Engineering Science 171 (2017) 638-649.

[19] K. Bäumler, M. Wegener, A.R. Paschedag, E. Bänsch, Drop rise velocities and fluid dynamic behavior in standard test systems for liquid/liquid extraction—experimental and numerical investigations, Chemical Engineering Science 66 (2011) 426-439. 
[20] J.R. Grace, T. Wairegi, T.H. Nguyen, Shapes and velocities of single drops and bubbles moving freely through immiscible liquids, Transactions of the Institution of Chemical Engineers 54 (1976) 167-173.

[21] J. Canny, A computational approach to edge detection, IEEE Transactions on Pattern Analysis and Machine Intelligence 6 (1986) 679-698.

[22] J. Westerweel, F. Scarano, Universal outlier detection for PIV data, Experiments in Fluids 39 (2005) 1096-1100.

[23] J.C. Agüí, J. Jiménez, On the performance of particle tracking, Journal of Fluid Mechanics 185 (1987) 447-468.

[24] C.A. Coulaloglou, L.L. Tavlarides, Description of interaction processes in agitated liquid liquid dispersions, Chemical Engineering Science 32 (1977) 1289-1297.

[25] H. Luo, H.F. Svenden, Theoretical model for drop and bubble breakup in turbulent dispersions, AIChE Journal 42 (1996) 1225-1233.

[26] J. Sheng, H. Meng, R.O. Fox, A large eddy PIV method for turbulence dissipation rate estimation, Chemical Engineering Science 55 (2000) 4423-4434.

[27] G. Bertens, D. van der Voort, H. Bocanegra-Evans, W. van de Water, Large-eddy estimate of the turbulent dissipation rate using PIV, Experiments in Fluids 56:89 (2015).

[28] C. Meneveau, T.S. Lund, The dynamic Smagorinsky model and scale-dependent coefficients in the viscous range of turbulence, Physics of Fluids 9 (1997) 3932-3934.

[29] S. Baldi, M. Yianneskis, On the quantification of energy dissipation in the impeller stream of a stirred vessel from fluctuating velocity gradient measurements, Chemical Engineering Science 59 (2004) 2659-2671.

[30] K.V. Sharp, R.J. Adrian, PIV study of small-scale flow structure around a Rushton turbine, AIChE Journal 47 (2001) 766-778.

[31] E.D. Hollander, J.J. Derksen, L.M. Portela, H.E.A. Van den Akker, Numerical scale-up study for orthokinetic agglomeration in stirred vessels, AIChE Journal 47 (2001) 2425-2440.

[32] R.S. Volkov, G.V. Kuznetsov, P.A. Strizhak, Water droplet deformation in gas stream: Impact of temperature difference between liquid and gas, International Journal of Heat and Mass Transfer 85 (2015) 1-11.

[33] R.S. Volkov, G.V. Kuznetsov, P.A. Kuibin, P.A. Strizhak, Features of water droplet deformation during motion in a gaseous medium under conditions of moderate and high temperatures, High Temperature 54 (2016) 722-730.

[34] J. Solsvik, S. Maaß, H.A. Jakobsen, Definition of the single drop breakup event, Industrial \& Engineering Chemistry Research 55 (2016) 2872-2882.

[35] Y. Liao, D. Lucas, A literature review of theoretical models for drop and bubble breakup in turbulent dispersions, Chemical Engineering Science 64 (2009) 3389-3406.

[36] N. Ninimiya, K. Yasuda, Visualization and PIV measurement of the flow around and inside of a falling droplet, Journal of Visualization 9 (2006) 257-264.

[37] S.V. Gupta, Measurement Uncertainties: Physical Parameters and Calibration of Instruments, Springer-Verlag, Berlin Heidelberg, 2012. 
Table 1

Table 1. Physical parameters of the binary systems silicone oil/water at $20{ }^{\circ} \mathrm{C}$ and toluene/water at $25{ }^{\circ} \mathrm{C}$.

\begin{tabular}{lcccccc}
\hline & $T /\left({ }^{\circ} \mathrm{C}\right)$ & $\rho /\left(\mathrm{kg} / \mathrm{m}^{3}\right)$ & $\mu /(\mathrm{mPa} \cdot \mathrm{s})$ & $\gamma /(\mathrm{mN} / \mathrm{m})$ & $M /(-)$ & Data source \\
& & & & & \\
\hline Silicone oil $(\mathrm{D})$ & 20 & 837 & 2.461 & 35.77 & $3.47 \times 10^{-11}$ & This study \\
Water $_{(\mathrm{C})}$ & & 998 & 1.000 & & \\
\hline Toluene $_{(\mathrm{D})}$ & 25 & 862 & 0.548 & 31.52 & $2.59 \times 10^{-11}$ & This study \\
Water $_{(\mathrm{C})}$ & & 997 & 0.883 & & & Bäumler et al. [19] \\
Toluene $_{(\mathrm{D})}$ & 25 & 862.3 & 0.552 & 35 & $1.95 \times 10^{-11}$ & \\
$\operatorname{Water}_{(\mathrm{C})}$ & 997.02 & 0.8903 & & & \\
\end{tabular}




\section{Table 2}

Table 2. The diameters $\left(d_{\mathrm{D}}\right)$, terminal velocities $\left(u_{\mathrm{D}}\right)$ and nondimensional parameters $\left(E o, R e_{\mathrm{D}}, M\right)$ of the silicone oil drops in the single drop experiments.

\begin{tabular}{cccccc}
\hline$d_{\mathrm{D}} /(\mathrm{mm})$ & $u_{\mathrm{D}} /(\mathrm{mm} / \mathrm{s})$ & $E o$ & $R e_{\mathrm{D}}$ & $M$ & $\log _{10}(M)$ \\
\hline 2.16 & 70.2 & 0.206 & 151 & $3.55 \times 10^{-11}$ & -10.45 \\
2.29 & 73.4 & 0.232 & 168 & & \\
2.31 & 75.4 & 0.236 & 174 & & \\
2.43 & 78.5 & 0.261 & 190 & & \\
2.48 & 80.1 & 0.272 & 198 & & \\
2.56 & 83.6 & 0.289 & 214 & & \\
2.77 & 87.1 & 0.339 & 241 & & \\
2.83 & 89.4 & 0.354 & 252 & & \\
2.89 & 90.1 & 0.369 & 260 & & \\
2.95 & 91.2 & 0.384 & 269 & & \\
3.17 & 99.0 & 0.444 & 313 & \\
3.27 & 100.5 & 0.472 & 328 & \\
3.83 & 113.2 & 0.648 & 433 & \\
\hline
\end{tabular}




\section{Table 3}

Table 3. The diameters $\left(d_{\mathrm{D}}\right)$, terminal velocities $\left(u_{\mathrm{D}}\right)$ and nondimensional parameters $\left(E o, R e_{\mathrm{D}}, M\right)$ of the toluene drops in the single drop experiments.

\begin{tabular}{cccccc}
\hline$d_{\mathrm{D}} /(\mathrm{mm})$ & $u_{\mathrm{D}} /(\mathrm{mm} / \mathrm{s})$ & $E o$ & $R e_{\mathrm{D}}$ & $M$ & $\log _{10}(M)$ \\
\hline 1.50 & 44.7 & 0.085 & 75 & $2.59 \times 10^{-11}$ & -10.59 \\
1.71 & 50.6 & 0.110 & 97 & & \\
1.83 & 55.6 & 0.127 & 114 & & \\
1.93 & 57.0 & 0.141 & 123 & & \\
2.19 & 65.2 & 0.181 & 160 & & \\
2.50 & 76.7 & 0.236 & 215 & & \\
3.01 & 87.0 & 0.342 & 293 & & \\
3.34 & 95.3 & 0.421 & 356 & & \\
3.58 & 96.2 & 0.484 & 386 & & \\
4.47 & 113.1 & 0.755 & 566 & & \\
\hline
\end{tabular}




\section{Table 4}

Table 4. The probability and time of drop breakup as a function of the jet Reynolds number Re. Num is the total number of drops per jet Reynolds number. $P_{1}$ is the probability of initial breakup at the first time, and $P_{2}$ is the probability of breakup cascade. Time $t_{1}$ is the time from the drop detaching from the needle to the first breakup, $t_{2}$ is the time from the drop detaching from the needle to the second breakup. The time ranges $t_{1}$ and $t_{2}$ indicate the shortest and longest time for the drop breakup.

\begin{tabular}{|c|c|c|c|c|c|c|}
\hline$R e$ & Num & $P_{1}$ & $P_{2}$ & $t_{1} /(\mathrm{s})$ & $t_{2} /(\mathrm{s})$ & \\
\hline 1283 & 16 & $0 \%$ & $0 \%$ & & & \\
\hline 1299 & 44 & $18 \%$ & & $0.18-0.19$ & & \\
\hline 1313 & 40 & $85 \%$ & $0 \%$ & $0.17-0.2$ & & Initial breakup \\
\hline 1323 & 20 & $100 \%$ & & $0.17-0.2$ & & \\
\hline 1333 & 20 & $95 \%$ & & $0.17-0.19$ & & \\
\hline 1349 & 24 & $96 \%$ & & $0.17-0.19$ & & \\
\hline 1372 & 20 & $95 \%$ & & $0.17-0.19$ & & \\
\hline 1391 & 20 & $100 \%$ & & $0.17-0.19$ & & \\
\hline 1414 & 20 & $100 \%$ & & $0.17-0.19$ & & \\
\hline 1447 & 24 & $96 \%$ & & $0.17-0.19$ & & \\
\hline 1476 & 20 & $100 \%$ & & $0.17-0.19$ & & \\
\hline 1501 & 23 & $100 \%$ & & $0.17-0.2$ & & \\
\hline 1536 & 24 & $79 \%$ & $17 \%$ & $0.18-0.21$ & $0.26-0.31$ & Breakup cascade \\
\hline 1560 & 43 & $70 \%$ & $23 \%$ & $0.18-0.21$ & $0.26-0.34$ & \\
\hline 1588 & 47 & $51 \%$ & $45 \%$ & $0.17-0.21$ & $0.27-0.35$ & \\
\hline 1610 & 20 & $10 \%$ & $0 \%$ & $0.19-0.20$ & & \\
\hline
\end{tabular}


Figure 1
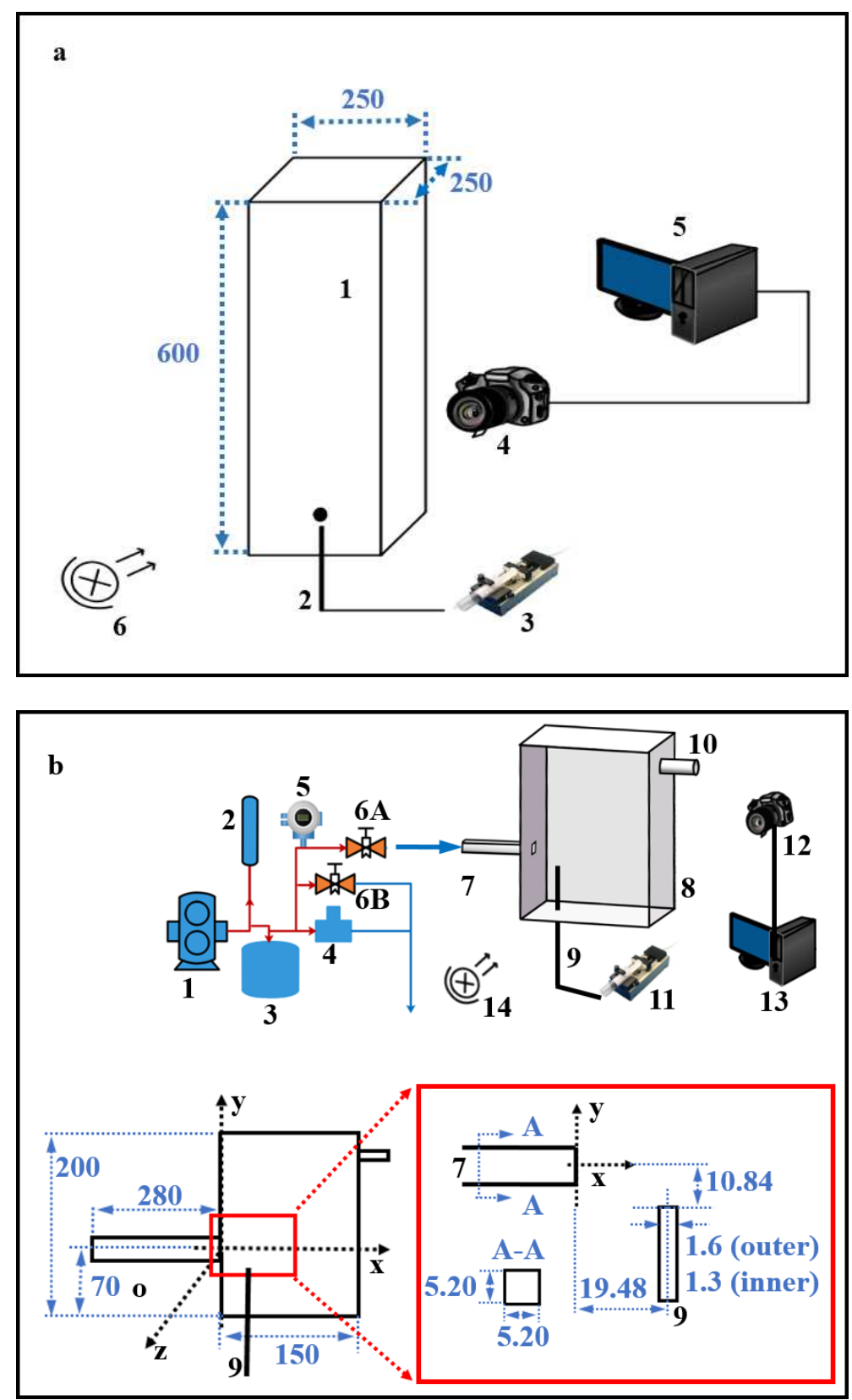

Fig. 1. (a) Experimental setup for drops rising in quiescent ambient water: 1) tank, 2) needle, 3) microinjection pump, 4) camera, 5) computer, 6) light. (b) Experimental setup for drop breakup in jet flow: 1) diaphragm pump, 2) damper, 3) buffer tank, 4) back pressure valve, 5) pressure gauge, 6) two needle valves, 7) jet flow pipe, 8) tank, 9) needle, 10) outlet, 11) microinjection pump, 12) camera, 13) computer, 14) light. Dimensions are in mm. 
Figure 2

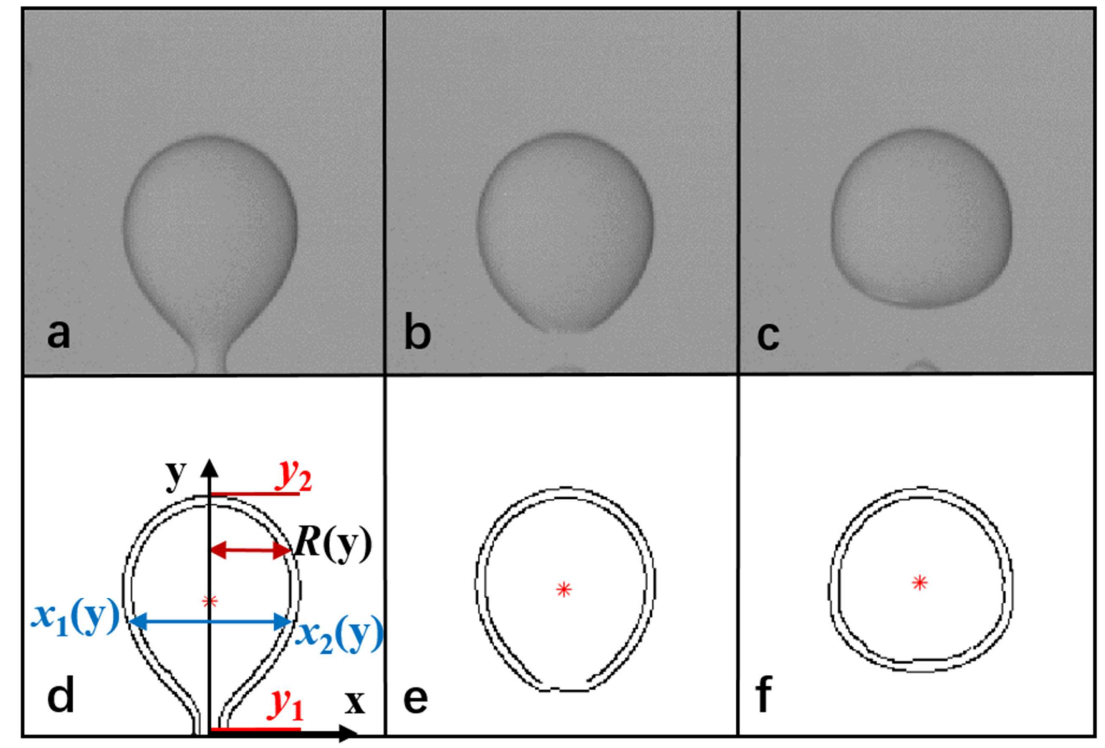

Fig. 2. Raw image of (a) a drop attached to the outlet of the needle and (b and c) shortly after detaching from the needle. The time separation between the images is $\Delta t=0.01 \mathrm{~s}$. Black lines in panels ( $\mathrm{d}$, e and $\mathrm{f}$ ) are the detected edges of the drops in panels ( $a, b$ and c) respectively. The red asterisks are the calculated centroids of the drop. In panel d, $y_{1}$ and $y_{2}$ are the highest and lowest positions of the drop, respectively; $x_{1}(y)$ and $x_{2}(y)$ are the left and right boundaries of the drop, respectively; $R$ is the radius of the drop as a function of $y$. 
Figure 3

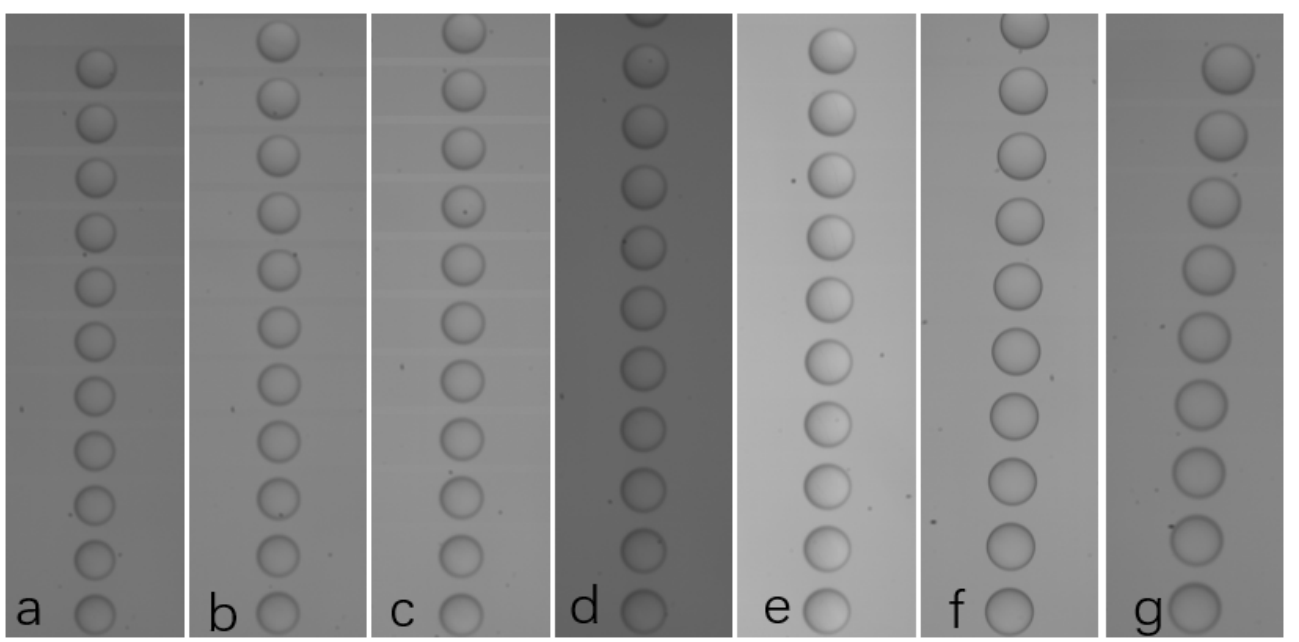

Fig. 3. Series of snapshots of silicon oil drops of different diameter rising in quiescent ambient water.

Drop diameters in panels (a)-(g) are 2.16, 2.29, 2.31, 2.43, 2.48, 2.56, and $2.77 \mathrm{~mm}$, respectively. $\Delta t=0.04 \mathrm{~s}$. The resolution is $0.01525 \mathrm{~mm} /$ pixel. 
Figure 4

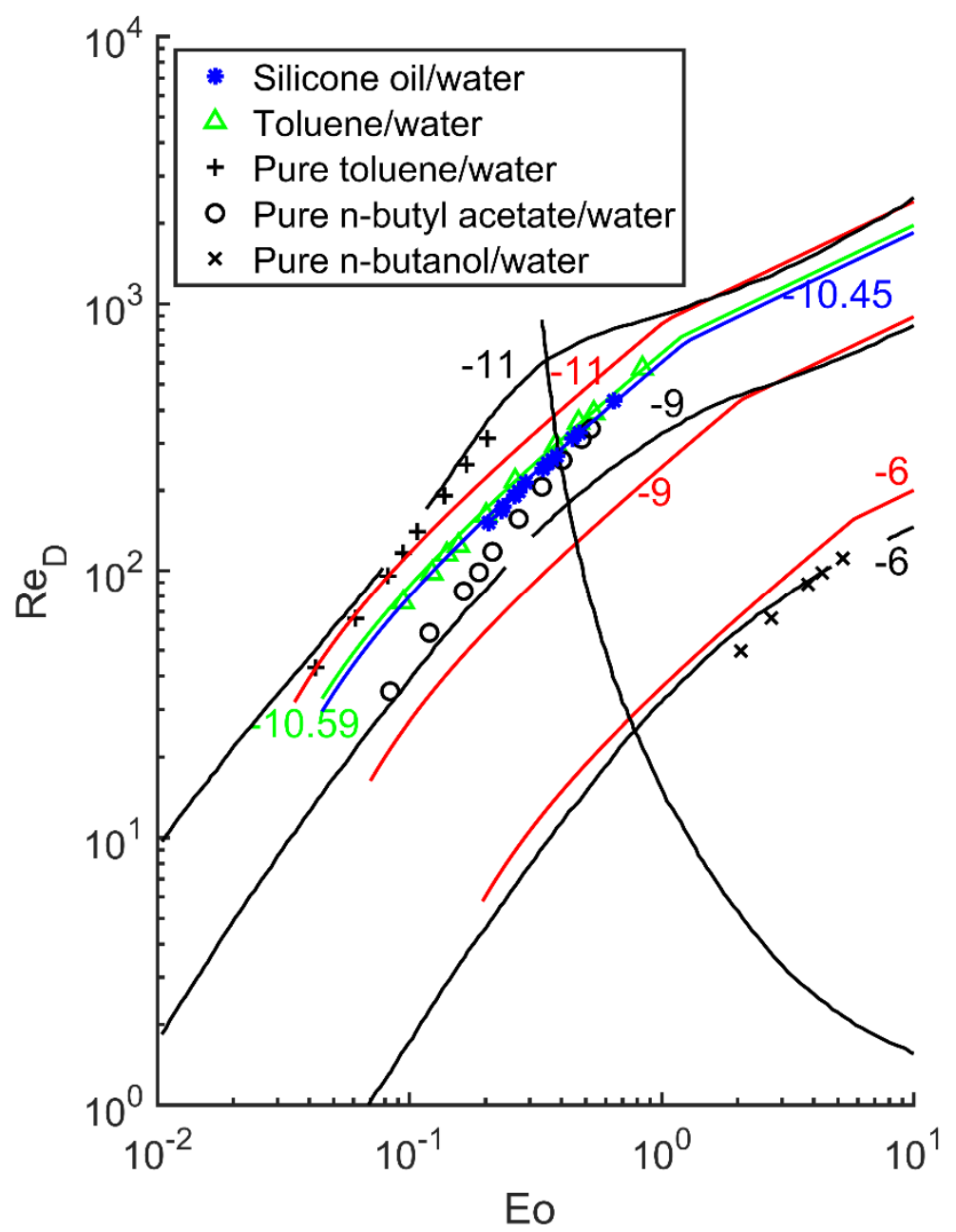

Fig. 4. Rising drop benchmark experiment results: Reynolds number $\left(R e_{\mathrm{D}}\right)$ as a function of Eötvös number $(E o)$ for certain Morton number $(M)$. The black solid lines are for pure system [20]; black symbols for pure toluene/water, pure n-butyl acetate/water, and pure n-butanol/water are the experimental data from Bäumler et al. [19]; the red, green, and blue lines are for contaminated systems [20]; the blue symbols are our experimental data for the silicone $\mathrm{oil} /$ water system, and green symbols are our experimental data for our toluene/water system. 
Figure 5

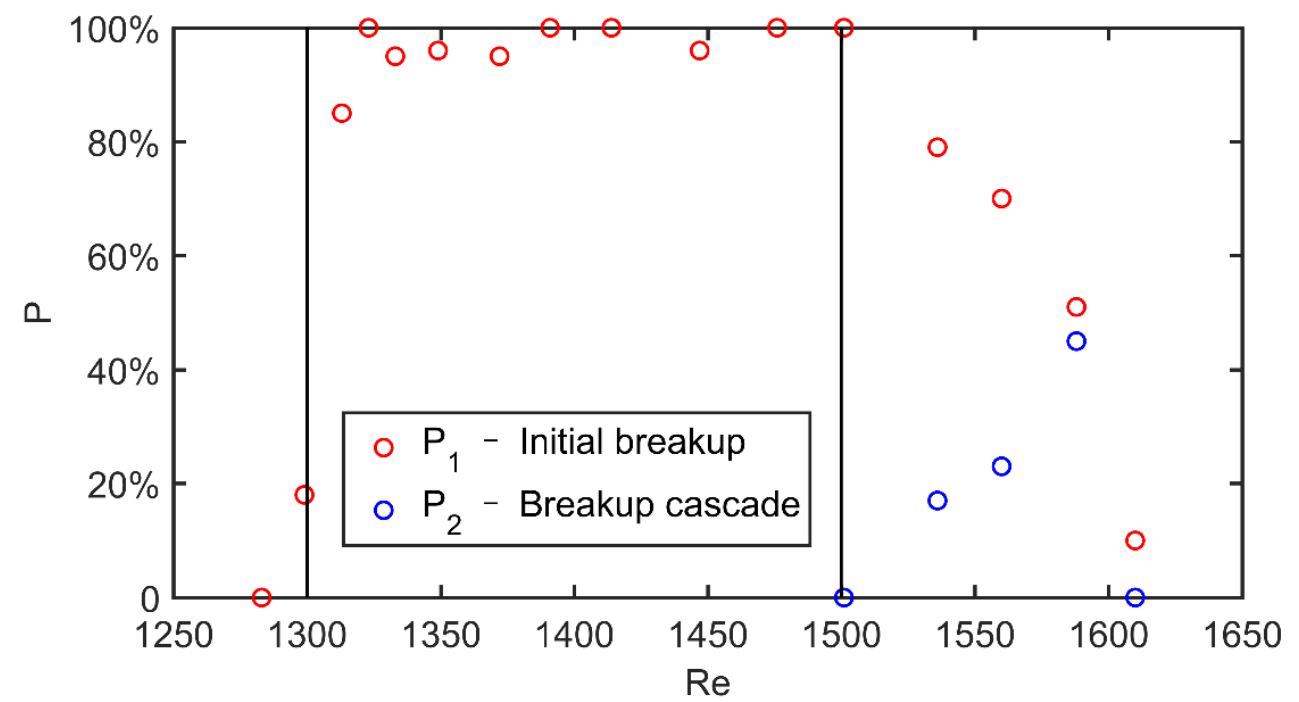

Fig. 5. The probability of the initial breakup and breakup cascade of oil drops in jet flow as a function of the jet Reynolds number $R e$. 


\section{Figure 6}

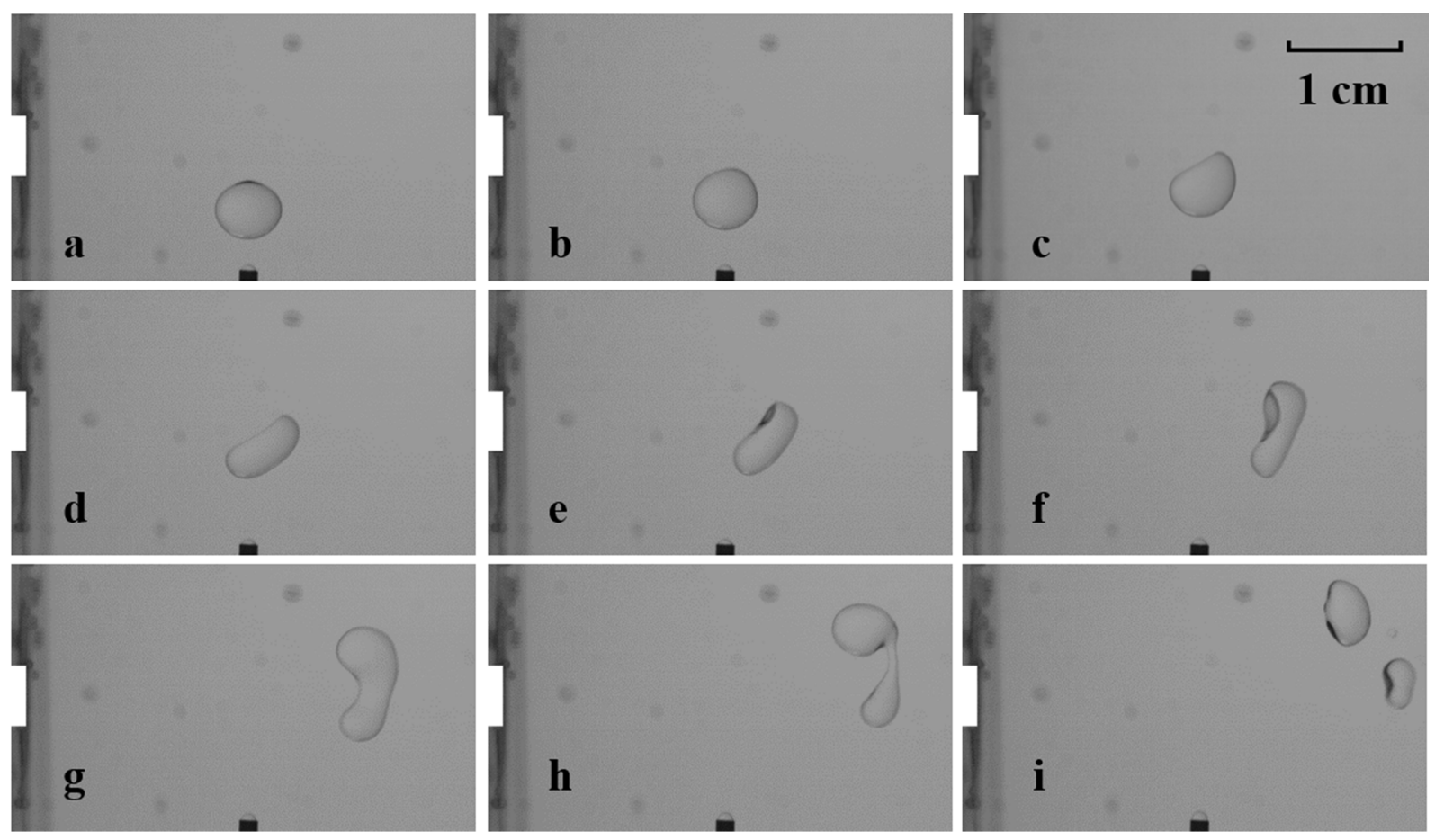

Fig. 6. $R e=1333$, the process of a drop breaking up into two daughters. The time interval between two successive drop images is $\Delta t=0.02 \mathrm{~s}$. The white area on the left is the exit of the jet pipe. 
Figure 7
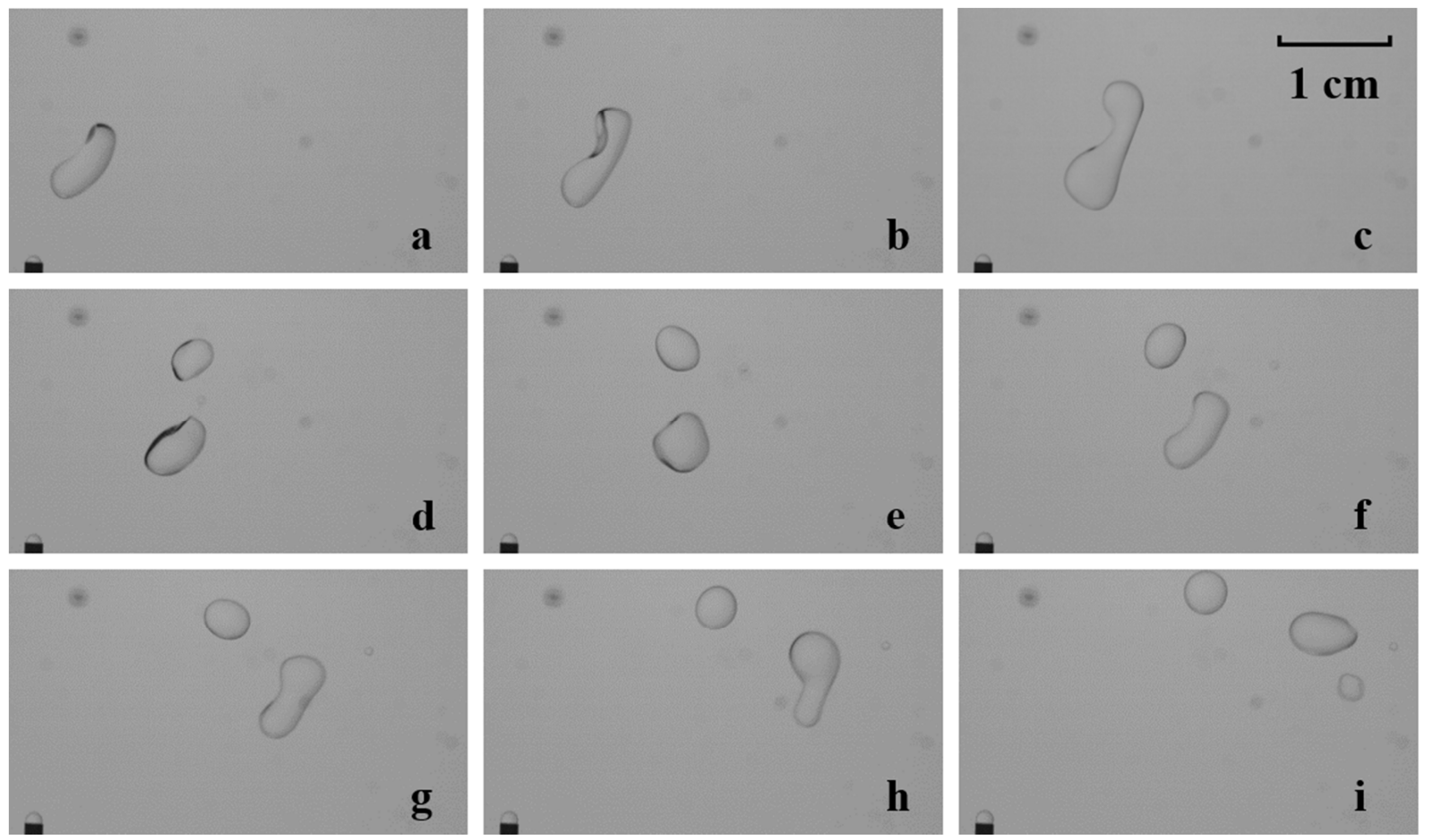

Fig. 7. $R e=1536$, the process of a breakup cascade.

The time interval between two successive drop images is $\Delta t=0.02 \mathrm{~s}$. 
Figure 8

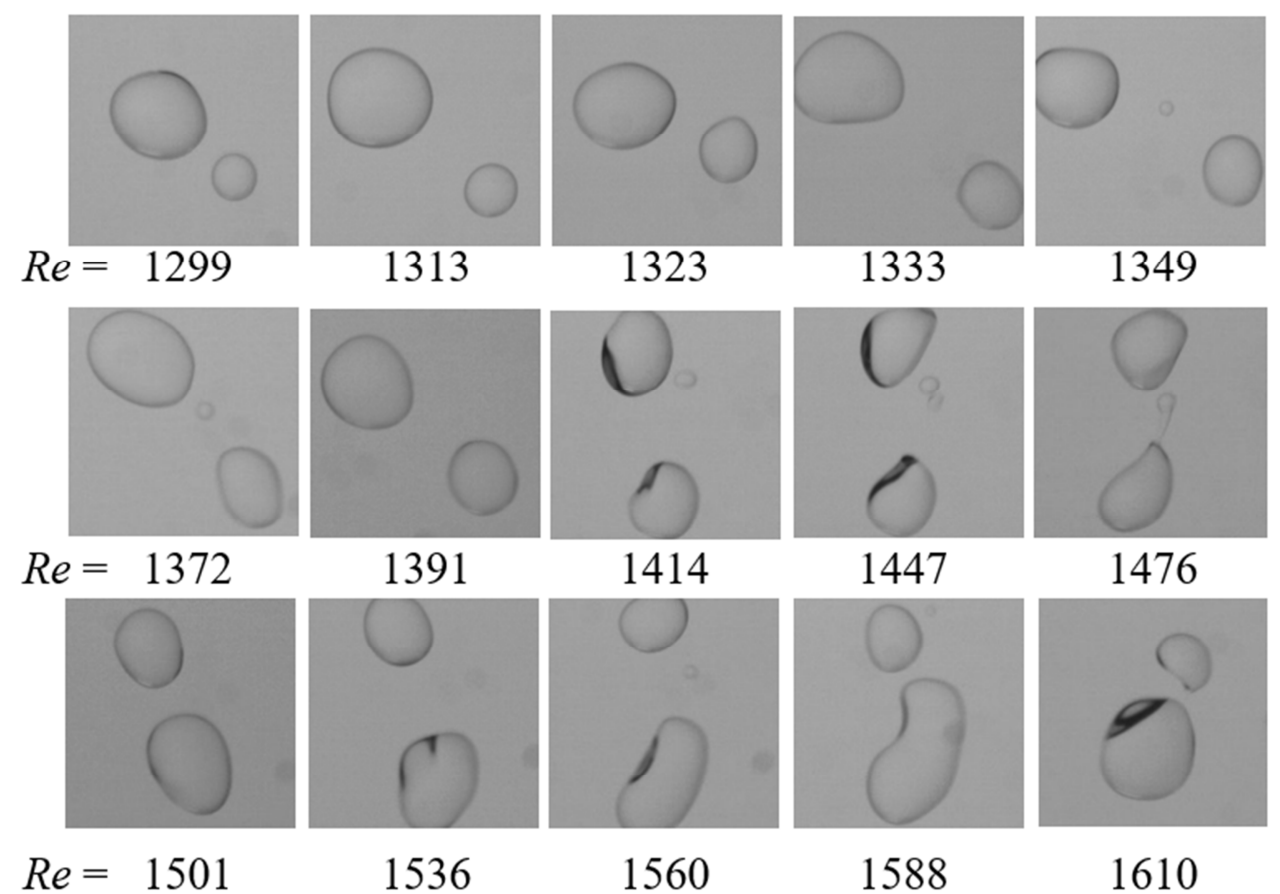

(a)

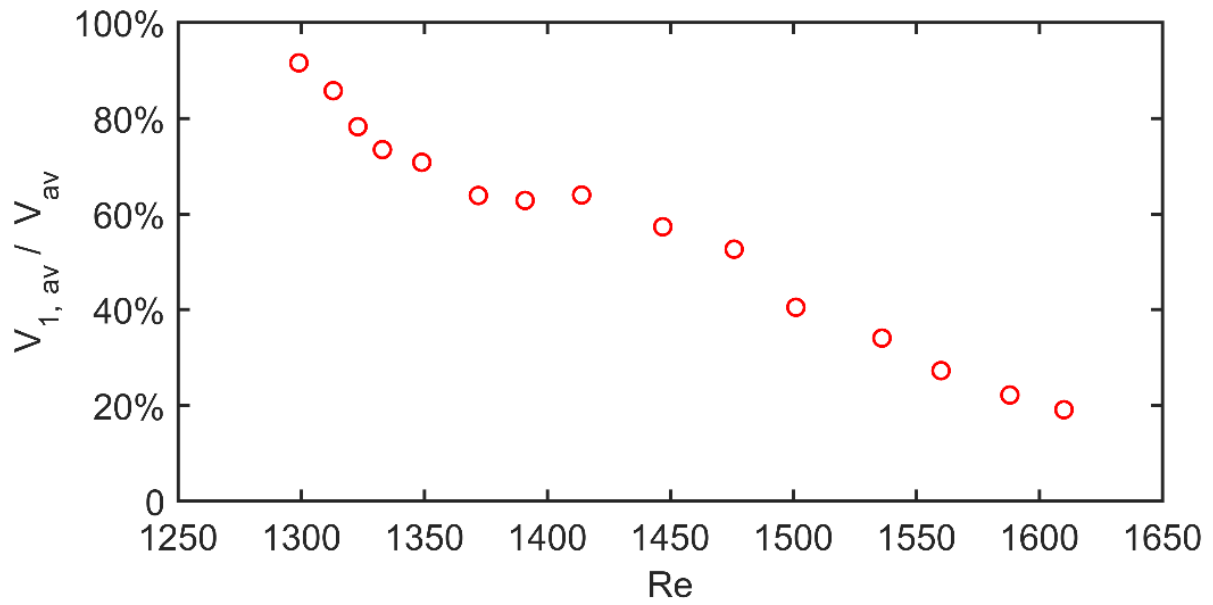

(b)

Fig. 8. (a) Daughter drops generated by initial breakup at various jet Reynolds numbers $R e$;

(b) Average volume of upper daughter drop to average volume of mother drop as a function of $R e$. 


\section{Figure 9}
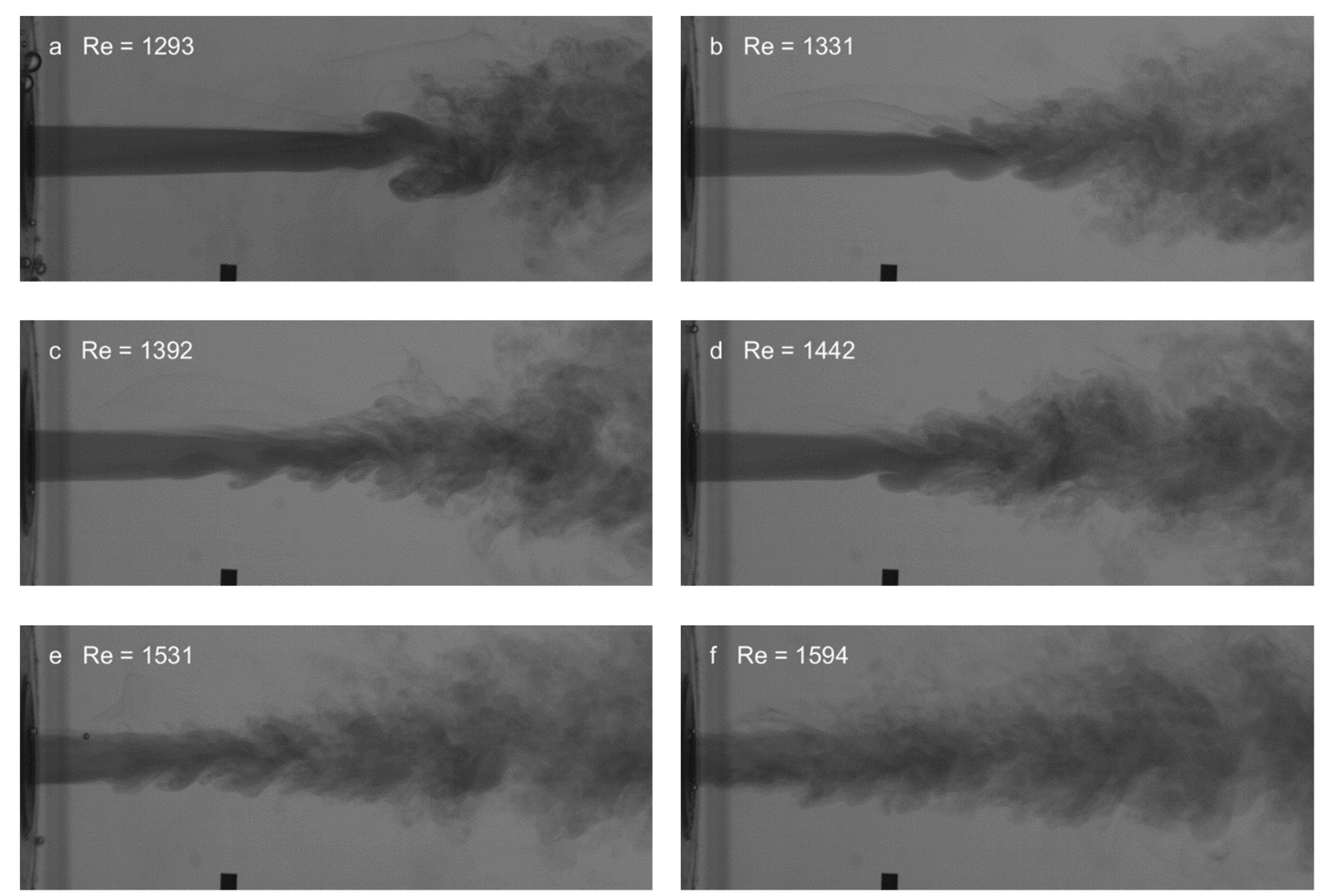

Fig. 9. Dye visualizations of the (single-phase) jet flow at various jet Reynolds numbers as indicated. The droplet release location is indicated by the black square near the bottom of each panel. 
Figure 10
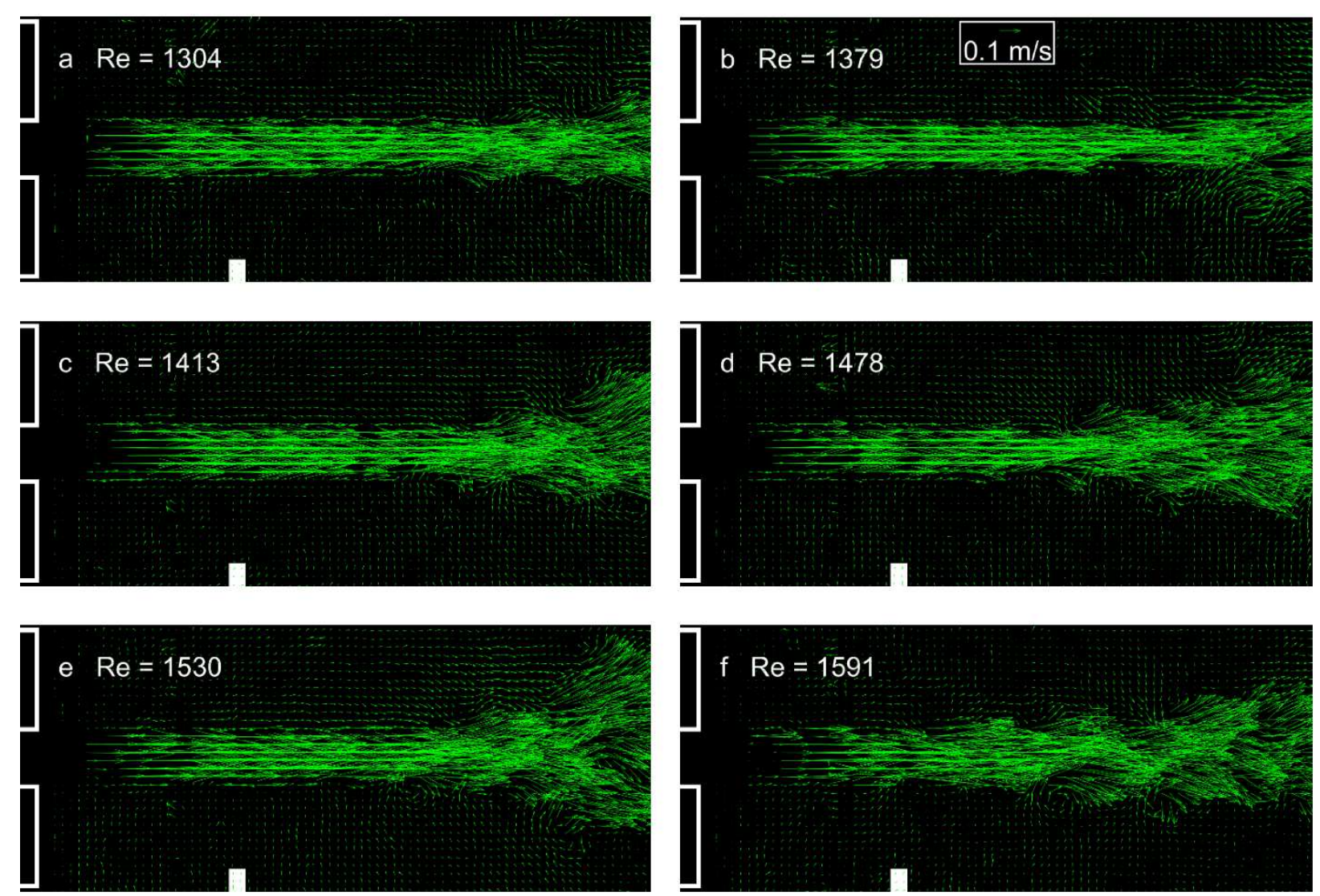

Fig. 10. Instantaneous velocity fields measured by 2-D PIV at different $R e$. The white lines on the left side are the edges of the jet flow pipe; the white square on the bottom is the needle releasing the drops. 


\section{Figure 11}
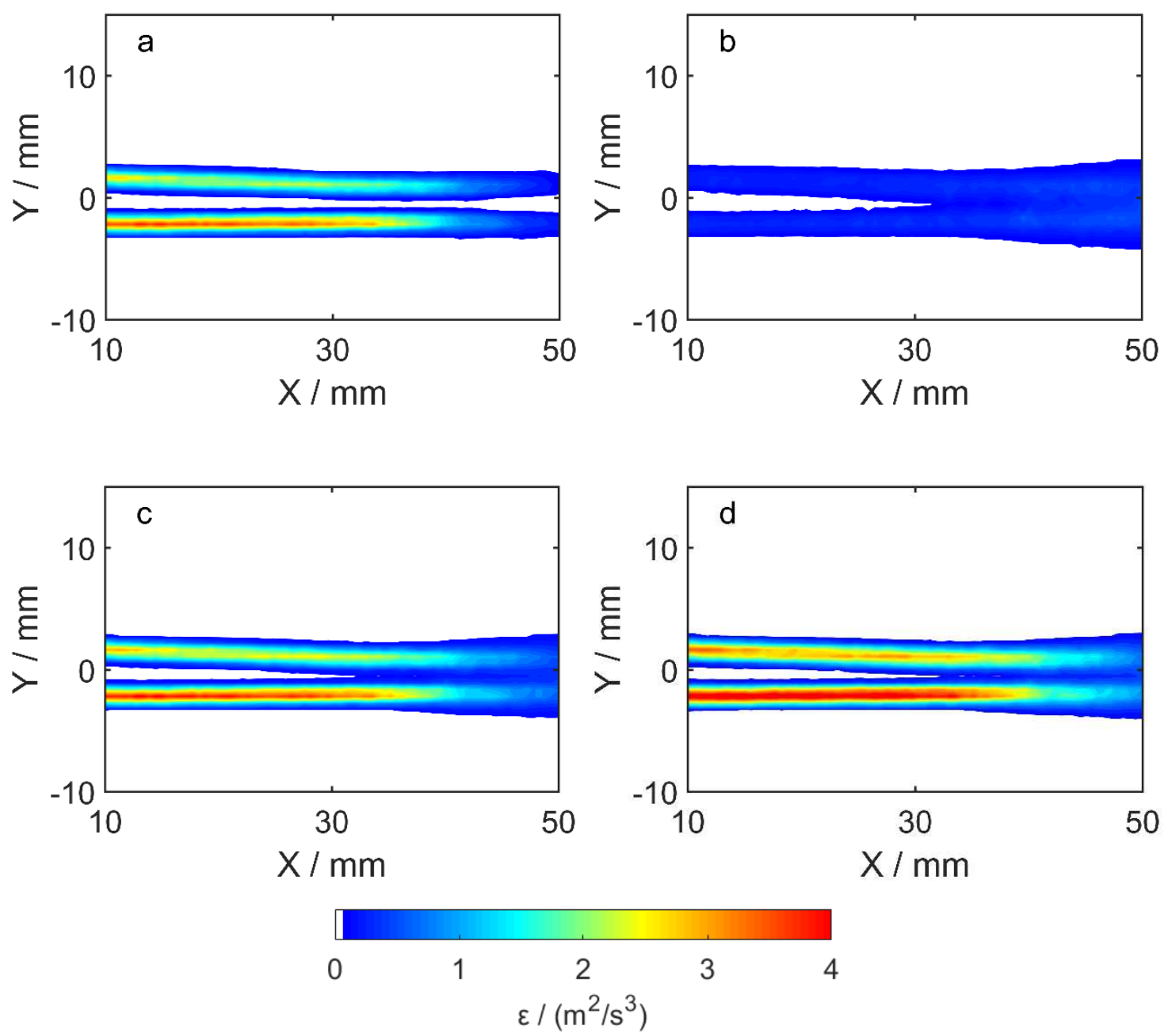

Fig. 11. Energy dissipation rate derived from PIV at $R e=1317$. (a): dissipation rate in the average flow $\langle\varepsilon\rangle_{\mathrm{av}}$; (b): in fluctuating flow $\langle\varepsilon\rangle_{\text {fluct }}$ (c): sum of average flow and fluctuating flow $\langle\varepsilon\rangle_{\mathrm{av}}+\langle\varepsilon\rangle_{\text {fluct }}$ (d): total dissipation calculated without performing a Reynolds decomposition $\langle\varepsilon\rangle_{\text {total }}$. 
Figure 12
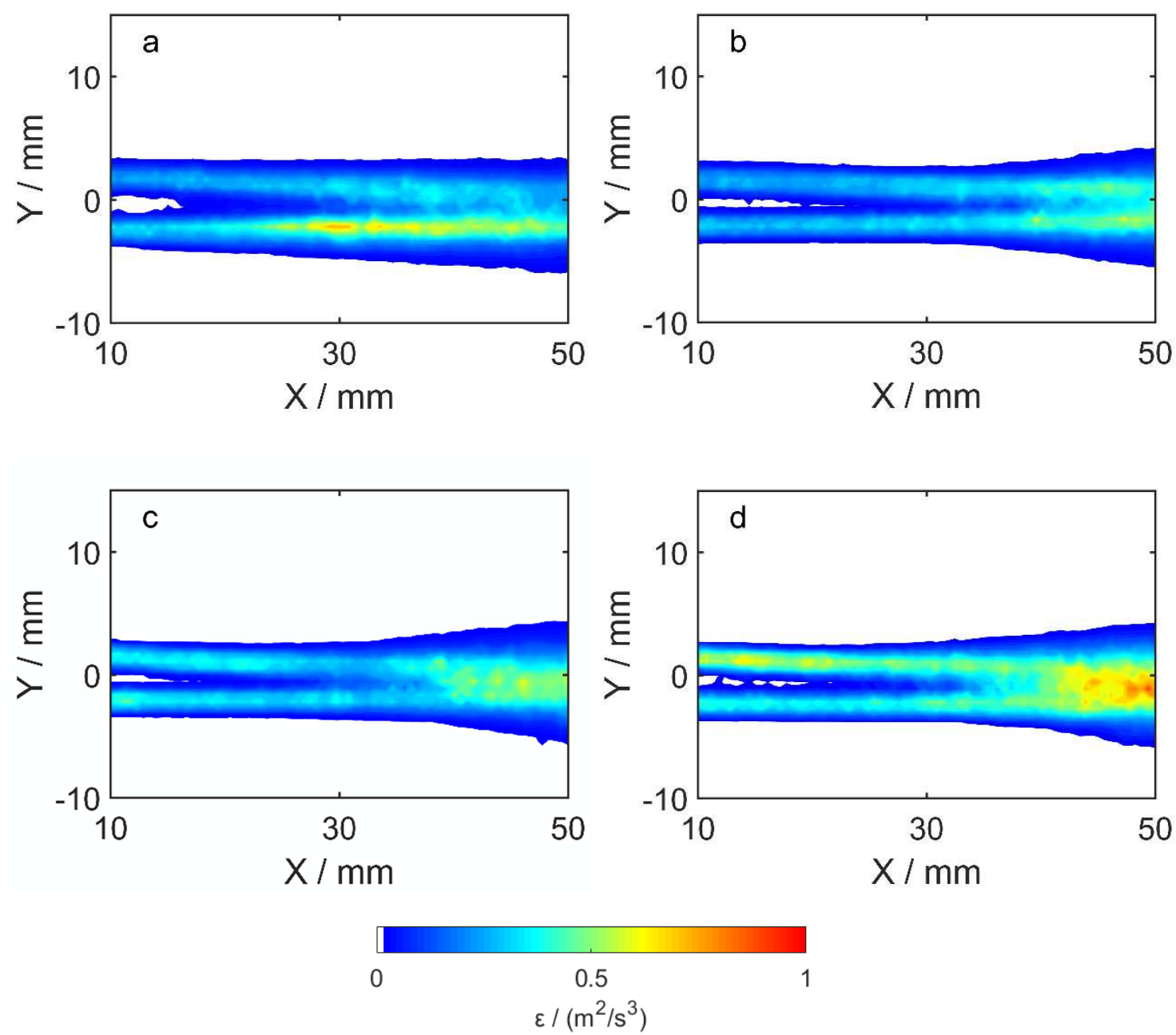

Fig. 12. The energy dissipation rate in the fluctuating flow $\langle\varepsilon\rangle_{\text {fluct }}$ at different Reynolds numbers. (a) $\operatorname{Re}=1286$; (b) $\operatorname{Re}=1317$; (c) $\operatorname{Re}=1456$; (d) $\operatorname{Re}=1584$. 


\section{Figure 13}
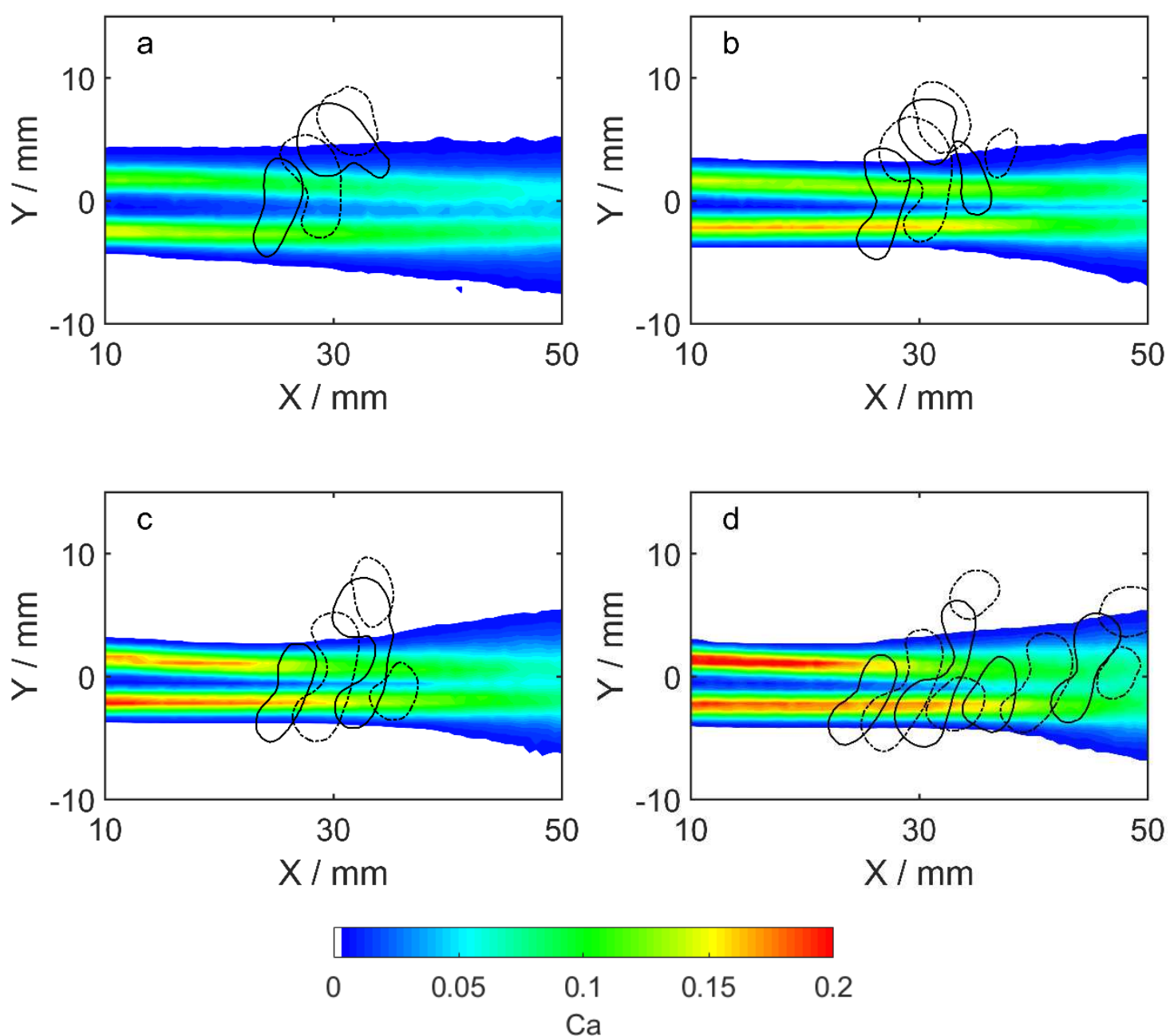

Fig. 13. Visualization of drop breakup combined with $\mathrm{Ca}$ contours derived from PIV at various jet Reynolds numbers.

The alternating solid and dot dash black lines indicate drop circumferences at moments $0.02 \mathrm{~s}$ apart.

(a) $R e_{1}=1283, R e_{2}=1286$; (b) $R e_{1}=1313, R e_{2}=1317$; (c) $R e_{1}=1447, R e_{2}=1456$; (d) $R e_{1}=1588, R e_{2}=1584$;

$R e_{1}$ and $R e_{2}$ are Reynold number in drops breakup experiments and in single-phase PIV experiments, respectively. 


\section{Figure 14}
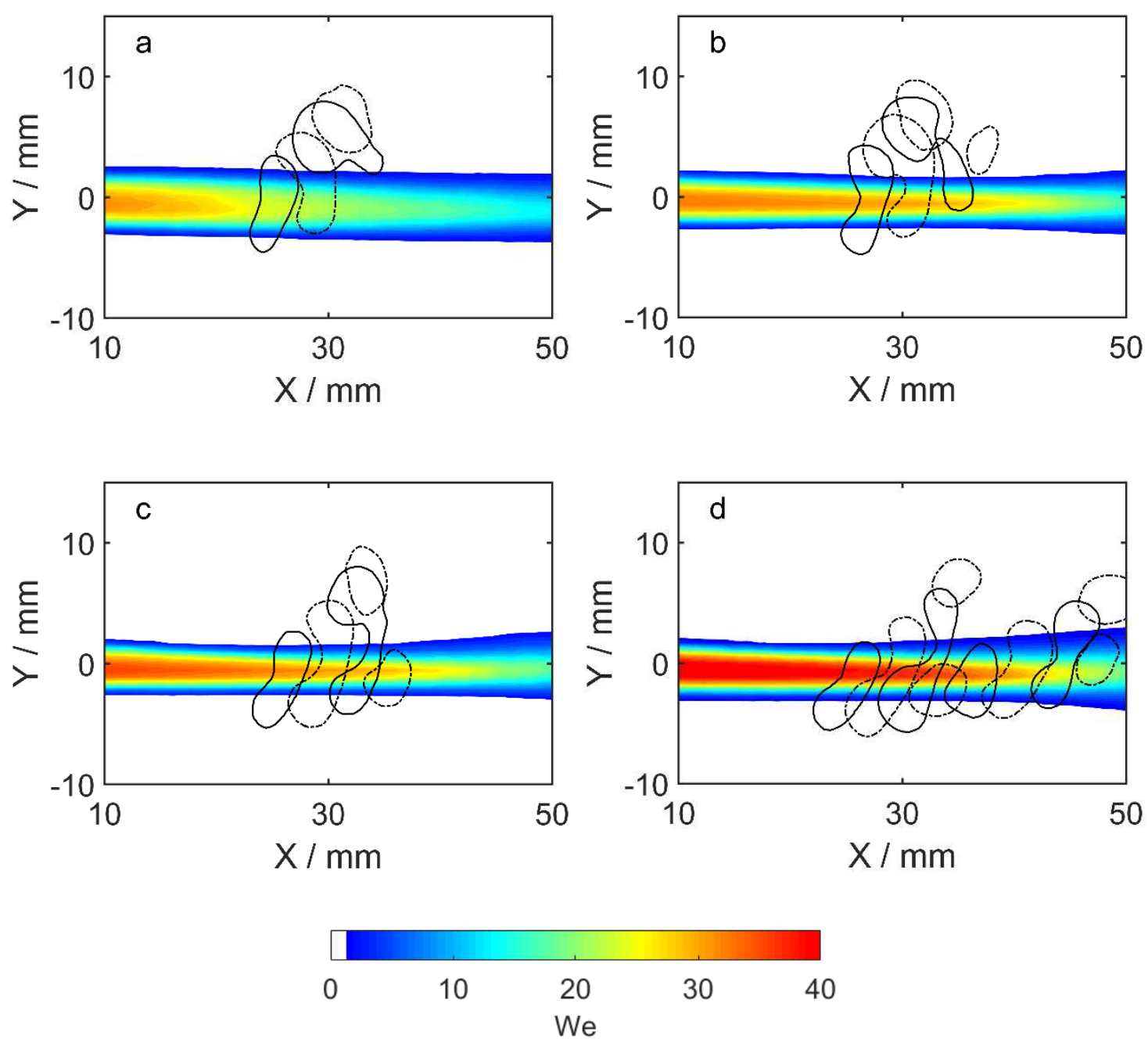

Fig. 14. Visualization of drop breakup combined with Weber number contours derived from PIV at various jet Reynolds numbers. The alternating solid and dot dash black lines indicate drop circumferences at moments $0.02 \mathrm{~s}$ apart. (a) $R e_{1}=1283, R e_{2}=1286$; (b) $R e_{1}=1313, R e_{2}=1317$; (c) $R e_{1}=1447, R e_{2}=1456$; (d) $R e_{1}=1588, R e_{2}=$ 1584; $R e_{1}$ and $R e_{2}$ are Reynold number in drops breakup experiments and in single-phase PIV experiments, respectively. 